\title{
THE TOPOLOGY OF RESOLUTION TOWERS
}

\author{
SELMAN AKBULUT AND HENRY KING
}

\begin{abstract}
An obstruction theory is given to determine when a space has a resolution tower. This can be used to decide whether or not the space is homeomorphic to a real algebraic set.
\end{abstract}

In $\left[\mathbf{A K}_{1}, \mathbf{A K}_{\mathbf{2}}\right]$ we have introduced topological resolution towers. These are certain topological structures on smooth stratified spaces. As the name suggests, such a structure gives a resolution of the space. Underlying topological spaces of real algebraic sets naturally have this structure $\left[\mathbf{A K}_{3}\right]$. In fact, a certain converse of this is also true $\left[\mathbf{A K}_{\mathbf{4}}\right]$. These structures impose new obstructions for a space to be a real algebraic set. In this paper we outline an obstruction theory for stratified spaces to admit these structures. This theory is similar to one suggested by Sullivan $[\mathbf{S}]$. As an application in $\left[\mathbf{A K}_{\mathbf{5}}\right.$ ] all real algebraic sets of dimension $\leqslant 3$ are topologically classified. The main theorem of this paper is Theorem 1.2, which is a generalization of our previous spine theorem (Proposition 4.2 of $\left[\mathbf{A} \mathbf{K}_{1}\right]$ ). Theorem 1.2 says that a stratified space $X$ with this structure bounds if and only if the structure on $X$ extends to cone $(X)$. This theorem makes the obstruction theory of this paper work.

Definitions. In this paper a manifold means a smooth manifold and a closed manifold means a compact smooth manifold without boundary. A proper codimension one submanifold $S$ of a closed manifold $V$ is called a separating submanifold if $S$ separates $V$. See Figure 1. If $A$ is a compact codimension zero submanifold of a compact manifold we define (see Figure 2)

$$
\begin{gathered}
\partial_{-}(A)=\partial(A) \cap \partial V, \\
\partial_{+}(A)=\partial(A)-\operatorname{int} \partial_{-}(A) .
\end{gathered}
$$

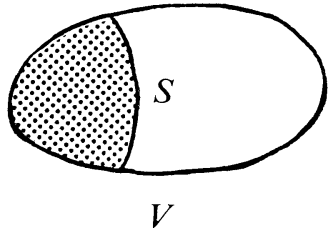

FIGURE 1

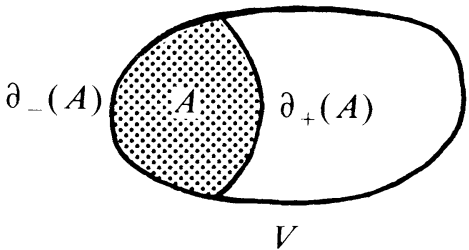

FigURE 2

Received by the editors September 12, 1985 and, in revised form, June 16, 1986. 1980 Mathematics Subject Classification (1985 Revision). Primary Primary 57R90, 14G30. Key words and phrases. Stratified, resolutions.

Sloan Fellows, also both authors supported in part by National Science Foundation. 


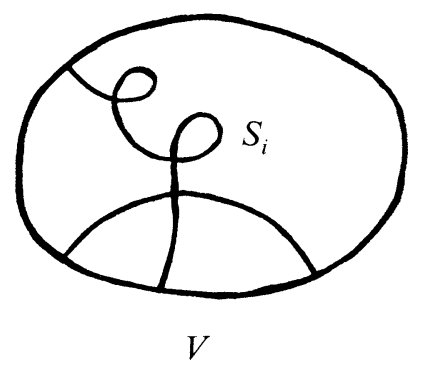

Figure 3

DEFINITION. Let $V$ be a compact manifold; then a tico $\mathfrak{A}$ in $V$ is a collection of properly immersed codimension one compact submanifolds of $V$ in general position meeting $\partial V$ transversally. Here proper means if $S \in \mathfrak{U}$ and $\partial S \neq \varnothing$, then $\partial S=$ $\partial V \cap S$. If $\mathfrak{U}=\left\{S_{i}\right\}_{i=1}^{n}$, each $S_{i}$ is called a sheet of $\mathfrak{A}$. We say $\mathfrak{A}$ is a regular tico if each $S_{i}$ is a properly imbedded submanifold. See Figure 3 . We define $\partial \mathfrak{A}=\left\{\partial S_{i}\right\}_{i=1}^{n}$; hence $\partial \mathfrak{U}$ is a tico in $\partial V$. Given ticos $\mathfrak{A}=\left\{S_{i}\right\}, \mathscr{B}=\left\{R_{j}\right\}$ in general position, define $\mathfrak{A} \cap \mathfrak{B}=\left\{S_{i} \cap R_{j}\right\}$. If $R$ is a smooth manifold which is in general position with all $S_{i}$, define $\mathfrak{U} \cap R=\left\{S_{i} \cap R\right\}$. If $L$ is a manifold define $\mathfrak{A} \times L=\left\{S_{i} \times L\right\}$, $\mathfrak{U} \times L$ is a tico in $V \times L$. We define the realization of a tico $\mathfrak{A}=\left\{S_{i}\right\}_{i=1}^{n}$ to be $|\mathfrak{A}|=\bigcup_{i=1}^{n} S_{i}$ (notice $\{|\mathfrak{A}|\}$ itself is a tico consisting of a single sheet).

A closed tico is a tico $\mathfrak{A}$ such that $\partial S=\varnothing$ for all $S \in \mathfrak{A}$. In particular, all ticos in a closed manifold are closed ticos. A separating tico is a regular tico such that each $S \in \mathfrak{A}$ is a separating submanifold of $V$. In this paper we use the symbol $\approx$ for stratified space isomorphism, so in particular if the stratified spaces are smooth manifolds, then $\approx$ denotes diffeomorphism. If $V$ is a compact manifold with boundary, we call a tico $\mathfrak{U}$ in $V$ a spine of $V$ if

(a) $\partial \mathfrak{U}=\varnothing$,

(b) $V$ is a regular neighborhood of $|\mathfrak{A}|$.

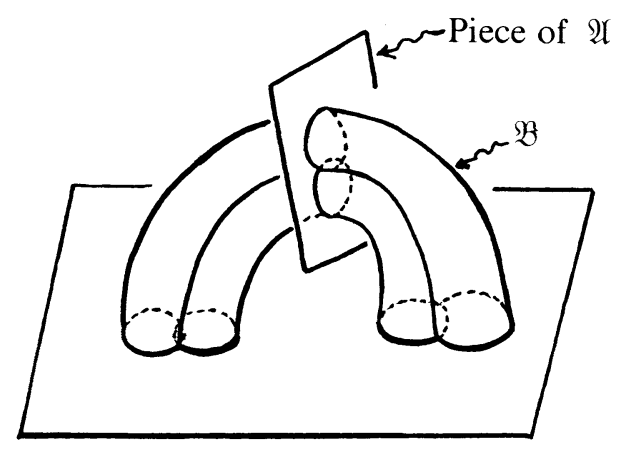

$V=$ the upper half space

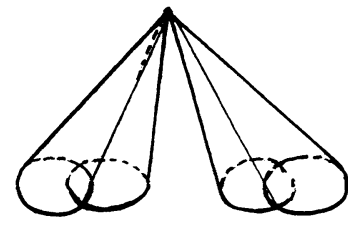

$|\mathfrak{B}| /|\mathfrak{A}| \cap|\mathscr{B}|$

FIGURE 4 
(b) implies that $V-|\mathfrak{A}| \approx \partial V \times(0,1]$, that is, $V /|\mathfrak{A}| \approx \operatorname{cone}(\partial V)$. If $\mathscr{U} \cup \mathfrak{B}$ is a tico in $V$ with $\partial \mathfrak{A}=\varnothing$ and $\partial \mathfrak{B} \neq \varnothing$, we say $\mathfrak{A}$ is a spine of $\mathfrak{B}$ if $|\mathfrak{B}| /|\mathfrak{A}| \cap|\mathfrak{B}| \approx$ cone $|\partial \mathfrak{B}|$. See Figure 4.

If $\mathfrak{A}=\left\{S_{i}\right\}_{i=1}^{n}$ is a tico then $|\mathfrak{A}|$ is a stratified space $\cup M_{j}$ in a natural way, where $M_{j}=\{x \in|\mathfrak{A}| \mid \alpha(x)=j\}$, and $\alpha(x)=\sum_{i=1}^{n} \alpha_{i}(x) . \alpha_{i}(x)$ is the multiplicity of $S_{i}$ at $x$. (Thus $\alpha_{i}(x)=0$ if $x \notin S_{i}, \alpha_{i}(x)=1$ if $S_{i}$ is imbedded near $x, \alpha_{i}(x)=2$ if $S_{i}$ has a double point at $x$, etc). In particular, if $\mathfrak{A}$ is regular then $\alpha(x)$ is the number of sheets $S_{i}$ passing through $x$ and $M_{j}$ is the set of points which are contained in exactly $j$ sheets. We also have a stratification on $V$, namely $V=(V-|\mathfrak{A}|) \cup \cup M_{j}$ which we will refer to as the stratification of $(V, \mathfrak{A})$.

In this paper sometimes we will have to consider a generalization of regular ticos, so we make the following definition.

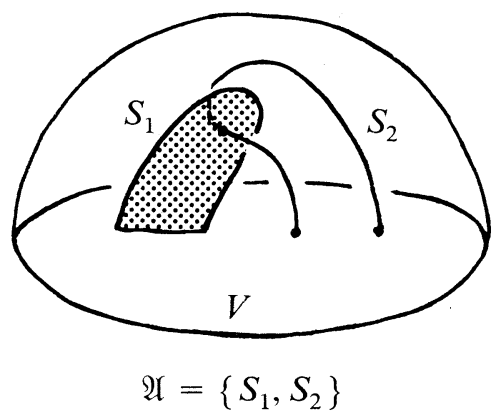

FIGURE 5

Definition. Let $V$ be a compact manifold. A tim $\mathfrak{A}$ in $V$ is a collection of transversally intersecting imbedded compact submanifolds of $V$, such that each $S \in \mathfrak{A}$ meets $\partial V$ transversally along a compact codimension zero submanifold of $\partial S$. We call the elements of $\mathfrak{A}$ sheets of $\mathfrak{A}$. Define $\left.\mathfrak{A}\right|_{\partial V}=\left\{\partial_{-} S\right\}_{S \in \mathfrak{A}},\left.\mathfrak{A}\right|_{\partial V}$ is a tim in $\partial V$. Define $|\mathfrak{A}|=\bigcup_{S \in \mathfrak{A}} S$, and call it the realization of tim $\mathfrak{A}$. Figure 5 gives a picture of a two-sheeted tim in the upper half ball.

Now we have to talk about several notions of transversality. Let $f: V \rightarrow W$ be a smooth map between smooth manifolds and let $\mathfrak{B}=\left\{S_{i}\right\}$ be a tim in $W$; then we say $f$ is transverse to $\mathfrak{B}$ if $f$ is transverse to all possible intersections of sheets of $\mathfrak{B}$. In this case we can pull back the tim $\mathfrak{B}$ to the tim $f^{*} \mathfrak{B}=\left\{f^{-1}\left(S_{i}\right)\right\}$ in $V$. Now in addition assume that $\mathfrak{A}$ is a tico in $V$; then we say $f: V \rightarrow W$ is tico-transverse to $\mathfrak{B}$ if the restriction of $f$ to every stratum of $V$ induced by $\mathfrak{A}$, is transverse to $\mathfrak{B}$. In this case the pull-back tim $f * \mathfrak{B}$ is transverse to $\mathfrak{A}$ in $V$. Similarly, we call a smooth map $f:|\mathfrak{A}| \rightarrow W$ tico-transverse to $\mathfrak{B}$ if the restriction of $f$ to every stratum of $|\mathfrak{A}|$ is transverse to $\mathfrak{B}$.

Definition. Let $\mathfrak{A}$ be a tico in a smooth manifold $V$. A subtim of $\mathfrak{U}$ is a collection $\mathfrak{E}$ of subsets of $|\mathfrak{A}|$ such that $\mathfrak{E}=\{B \cap|\mathfrak{A}| \mid B \in \mathfrak{B}\}$ for some tim $\mathfrak{B}$ in 


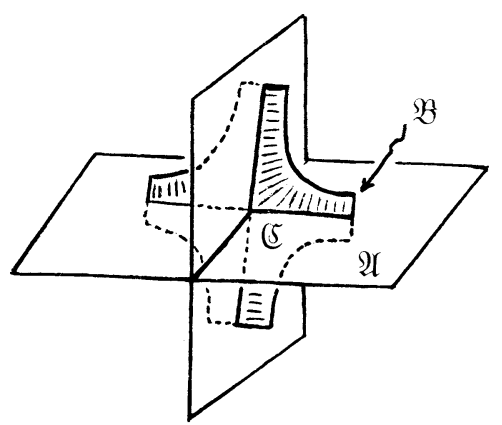

Figure 6

general position with $\mathfrak{A}$. See Figure 6. Note that a subtim is not itself a tim. The tim $\mathfrak{B}$ will be called a normal thickening of $\mathfrak{E}$. Note that normal thickenings are far from unique. The following lemma gives a useful criterion for when a subset of $|\mathfrak{A}|$ is a subtim. Let $g: N \rightarrow V$ be the immersion associated to $\mathfrak{A}$. That is, $g(N)=|\mathfrak{A}|$ and $g$ is one-to-one on a dense subset of $N$. In $\left[\mathbf{A K}_{2}\right]$, we defined a pull-back tico $g^{*}(\mathfrak{A})$ in $N$.

LEMma 1.1 (NORMAL THICKENING LEMMA). A finite collection 5 of subsets of $|\mathfrak{A}|$ is a subtim of $\mathfrak{A}$ if and only if $\mathfrak{D}=\left\{g^{-1}(C) \mid C \in \mathbb{C}\right\}$ is a tim in $N$ in general position with $g^{*}(\mathfrak{A})$. In case $\mathfrak{A}$ is regular this is equivalent to saying that for each sheet $S$ of $\mathfrak{A}$, $\{S \cap C \mid C \in \mathbb{E}\}$ is a tim in $S$ which is in general position with the tico $S \cap \mathfrak{U}=$ $\{S \cap A \mid A \in \mathfrak{A}-\{S\}\}$.

Proof. If $\mathbb{E}=\{|\mathfrak{A}| \cap B \mid B \in \mathfrak{B}\}$ for some tim $\mathfrak{B}$ in general position with $\mathfrak{A}$, then it is easy to check that $\mathscr{D}=\left\{g^{-1}(B) \mid B \in \mathfrak{B}\right\}$ is a tim in $N$ in general position with $g^{*}(\mathfrak{A})$. To prove the converse, suppose we know $\mathfrak{D}$ is a tim in $N$ transverse to $g^{*}(\mathfrak{U})$. By induction we may assume there is a tim $\mathfrak{B}^{\prime}$ in general position with $\mathfrak{A}$ and a $C \in \mathfrak{C}$ so that $\mathfrak{S} \cup\left\{\partial_{+} C\right\}-\{C\}=\left\{|\mathfrak{A}| \cap B \mid B \in \mathfrak{B}^{\prime}\right\}$, where $\partial_{+} C=$ $g\left(\partial_{+}\left(g^{-1}(C)\right)\right)$. Now for every $z \in C$ it is not hard to find a chart $\phi_{z}:\left(\mathbf{R}^{m}, 0\right) \rightarrow(V, z)$ (or $\phi_{z}:\left(\left\{x \in \mathbf{R}^{m} \mid x_{m} \geqslant 0\right\}, 0\right) \rightarrow(V, z)$ if $\left.z \in \partial V\right)$ so that $\phi_{z}^{-1}$ of everything in sight is linear. That is, $\phi_{z}^{-1}(|\mathfrak{A}|)=\bigcup_{i=1}^{a} \mathbf{R}_{i}^{m}$ (where $\mathbf{R}_{i}^{m}=\left\{x \in \mathbf{R}^{m} \mid x_{i}=0\right\}$ ) and for each $B \in \mathfrak{B}^{\prime}$ either $z \notin B$ or $\phi_{z}^{-1}(B)=\bigcap_{i=b}^{c} \mathbf{R}_{i}^{m} \cap$ (domain of $\phi_{z}$ ) (if $z \in B-\partial_{+} B$ ) or $\phi_{z}^{-1}(B)=\bigcap_{i=b}^{c-1} \mathbf{R}_{i}^{m} \cap\left\{x \in \mathbf{R}^{m} \mid x_{c} \geqslant 0\right\} \cap$ (domain of $\phi_{z}$ ) (if $z \in \partial_{+} B$ ) where $b$ and $c$ depend on $B$. Furthermore, there is some linear subspace $S_{z} \subset \mathbf{R}^{m}$ transverse to $\phi_{z}^{-1}(|\mathfrak{A}|)$ and a smooth $\theta_{z}: S_{z} \cap \phi_{z}^{-1}(|\mathfrak{A}|) \rightarrow S_{z}{ }^{\perp}$ so that

$$
\phi_{z}^{-1}(C)=\left\{x+\theta_{z}(x) \mid x \in S_{z} \cap \phi_{z}^{-1}(|\mathfrak{A}|)\right\}
$$

So by covering $C$ with a finite number of such charts and by extending each $\theta_{z}$ to a neighborhood $S_{z}^{\prime}$ of $S_{z} \cap \phi_{z}^{-1}(|\mathfrak{A}|)$ in $S_{z} \cap$ domain $\phi_{z}$ we may find some manifold $B^{\prime} \subset V$ so that $\phi_{z}^{-1}\left(B^{\prime}\right)=\left\{x+\theta_{z}(x) \mid x \in S_{z}^{\prime}\right\}$. Then $\mathscr{B}=\mathscr{B}^{\prime} \cup\left\{B^{\prime}\right\}-\left\{\partial_{+} B^{\prime}\right\}$ is a tim in general position with $\mathfrak{A}$ and $\mathfrak{E}=\{|\mathfrak{A}| \cap B \mid B \in \mathfrak{B}\}$. 


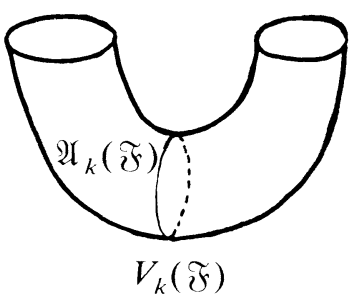

FIGURE 7

Definition. Let $(V, \mathfrak{A}),(W, \mathfrak{B})$ be two closed smooth manifolds with ticos. A smooth map $f: V \rightarrow W$ is called a tico map if the following is true. for any $q \in V$ and $f(q) \in W$ pick charts $\psi:\left(\mathbf{R}^{m}, 0\right) \rightarrow(V, q), \theta:\left(\mathbf{R}^{n}, 0\right) \rightarrow(W, f(q))$ so that $\psi^{-1}(|\mathfrak{A}|)=\bigcup_{i=1}^{a} \mathbf{R}_{i}^{m}, \theta^{-1}(|\mathfrak{B}|)=\bigcup_{i=1}^{b} \mathbf{R}_{i}^{n}$ and $f \psi\left(\mathbf{R}^{m}\right) \subset \theta\left(\mathbf{R}^{n}\right)$. Let $k_{i}(x)$ be the $i$ th coordinate of $\theta^{-1} f \psi(x)$. Then $f$ is a tico map if there are nonnegative integers $\alpha_{i j}$, $1 \leqslant i \leqslant b, \quad 1 \leqslant j \leqslant a$ and smooth functions $\phi_{i}: \mathbf{R}^{m} \rightarrow \mathbf{R}$ such that $k_{i}(x)=$ $\prod_{j=1}^{a} x_{j}^{\alpha_{i j}} \phi_{i}(x)$ for all $x$ near 0 and $\phi_{i}(0) \neq 0$ for $i=1,2, \ldots, b$. Let $\mathfrak{C} \subset \mathfrak{A}$; then we say a map $g:|\mathfrak{S}| \rightarrow W$ is a mico if there is a neighborhood $U$ of $|\Subset|$ in $V$ and a tico map $f:(U, U \cap \mathfrak{A}) \rightarrow(W, \mathfrak{B})$ such that $g=f \mid$. Similarly we can define ticos and micos in case $V$ and $W$ are not closed by allowing coordinate charts from upper half Euclidean spaces.

We are now ready to define resolution towers, which is the basic object of this paper. A resolution tower is a collection $\mathfrak{F}=\left\{V_{i}, \mathfrak{A}_{i}, p_{i}\right\}_{i=0}^{n}$ where $\left(V_{i}, \mathfrak{A}_{i}\right), i=$ $0, \ldots, n$, are compact smooth manifolds with ticos and $p_{i}=\left\{p_{j i}\right\}_{j=0}^{i-1}$ are collections of smooth maps $p_{j i}: V_{j i} \rightarrow V_{j}$ so that $V_{j i}=\left|\mathfrak{A}_{j i}\right|$ for some $\mathfrak{U}_{j i} \subset \mathfrak{U}_{i}$ and

(1) $p_{j i}\left(V_{j i} \cap V_{k i}\right) \subset V_{k j}$ for $k<j<i$,

(2) $\left.p_{k j} \circ p_{j i}\right|_{v_{i i}} \cap v_{k i}=\left.p_{k i}\right|_{v_{j i} \cap V_{k i}}$,

(3) $p_{j i}^{-1}\left(\bigcup_{k<m} V_{k j}\right)=V_{j i} \cap\left(\bigcup_{k<m} V_{k i}\right)$,

(4) $\mathfrak{A}_{i}=\bigcup_{j<i} \mathfrak{A}_{j i}$, and $\mathfrak{A}_{j i} \cap \mathfrak{A}_{k i}=\varnothing$ when $j \neq k$,

(5) $p_{j i}^{-1}\left(\partial V_{j}\right)=\partial V_{i} \cap V_{j i}$.

A resolution tower is a purely topologically defined object; to emphasize this, we will sometimes call it a topological resolution tower. We will denote $V_{i}=V_{i}(\mathfrak{F})$, $\mathfrak{U}_{i}=\mathfrak{U}_{i}(\mathfrak{F}), \quad \mathfrak{A}_{j i}=\mathfrak{U}_{j i}(\mathfrak{F}), \quad V_{j i}=V_{j i}(\mathfrak{F}), \quad$ and $\quad p_{j i}=p_{j i}(\mathfrak{F})$. We define $n=$ height $(\mathfrak{F})$. We call a topological resolution tower $\mathfrak{F}$ seminormal (normal) if whenever $W$ is any component of $V_{i}(\mathfrak{F})$ with $\partial W \neq \varnothing$ and $U$ is a component of $W-\left|\mathfrak{A}_{i}(\mathfrak{F})\right|$, then $U \cap \partial W \neq \varnothing(U$ meets all the components of $\partial W)$. Clearly normal implies seminormal but not the converse; for example, Figure 7 satisfies the seminormal condition but not the normality condition. In case all $\partial V_{i}=\varnothing$ seminormality and normality are vacuous conditions.

We can talk about some additional properties of resolution towers (see $\left[\mathbf{A} \mathbf{K}_{2}\right]$ ); the ones we use in this paper are:

$R$-Each tico $\mathfrak{A}_{i}$ is regular.

$M-$ Each $p_{j i}$ is a mico.

$U$-Each $\left.p_{j i}\right|_{S}$ is a smooth submersion for every stratum $S$ of $\left(V_{i}, \mathfrak{A}_{i}\right)$ with $S \subset V_{j i}-\cup_{k<j} V_{k i}$. 
$S$-Each $p_{j i}$ is a submersive mico, in particular $\left.p_{j i}\right|_{S}$ is a smooth submersion for every stratum $S$ of $\left(V_{i}, \mathfrak{U}_{i}\right)$ (see $\left[\mathbf{A K}_{2}\right]$ ).

$F$-Each $\left(V_{i}, \mathfrak{A}_{i}\right)$ is full. This means that if $S$ is $V_{i}$ or any intersection of sheets of $\mathfrak{A}_{i}, H_{*}(S ; \mathbb{Z} / 2 \mathbb{Z})$ is generated by imbedded submanifolds of $S$.

For example, we will say $\mathfrak{F}$ is type $R$ it it satisfies $R$. Sometimes we will combine these properties; for instance, a resolution tower $\mathfrak{F}$ of type $R M$ satisfies both the properties $R$ and $M$.

If $\mathfrak{F}=\left\{V_{i}, \mathfrak{A}_{i}, p_{i}\right\}$ then we define $\partial \mathfrak{F}=\left\{\partial V_{i}, \partial \mathfrak{A}_{i}, p_{i} \mid\right\}$. We call $\mathfrak{F}$ closed if $\partial \mathscr{F}=\varnothing$. For $0 \leqslant k \leqslant n$ we denote $\mathfrak{F}_{k}=\left\{V_{i}, \mathfrak{A}_{i}, p_{i}\right\}_{i=0}^{k}$.

An algebraic resolution tower is a resolution tower such that each $V_{i}$ is a nonsingular algebraic set and each sheet of $\mathfrak{A}_{i}$ is a nonsingular algebraic subset and each $p_{j i}$ is an entire rational function. We define $\mathscr{T}$ to be the set of all topological resolution towers and $\mathscr{A}$ to be the set of all algebraic resolution towers. When we put subscripts $R, M, U, S, F$ to $\mathscr{T}$ or $\mathscr{A}$, they will denote the subsets of $\mathscr{T}$ or $\mathscr{A}$ of that type. For example, $\mathscr{T}_{R M}$ is the set of resolution towers of type $R M$. Clearly, we can identify $\mathscr{A} \hookrightarrow \mathscr{T}$ via the forgetful map. Note that $\mathscr{A}_{R}=\mathscr{A}$ and $\mathscr{T}_{S} \subset \mathscr{T}_{M U}$, $\mathscr{A}_{S} \subset \mathscr{A}_{M U}$.

If $\mathfrak{F} \in \mathscr{T}$ we define the realization of $\mathfrak{F}$ to be

$$
|\mathfrak{F}|=\bigcup_{i=0}^{n} V_{i} / x \sim p_{j i}(x) .
$$

That is, $|\mathfrak{F}|$ is obtained by identifying the points $x \sim p_{j i}(x)$ for all $x \in V_{j i}$ in the disjoint union $\bigcup_{i=0}^{n} V_{i}$. If $\mathfrak{F} \in \mathscr{T}$ then $|\mathfrak{F}|$ is a stratified space with strata $\left\{V_{j}-\right.$ $\left.\bigcup_{r<j} V_{r j}\right\}$. We define $\operatorname{dim}(\mathfrak{F})$ to be the dimension of $|\mathfrak{F}|$, i.e. the dimension of the highest dimensional stratum.

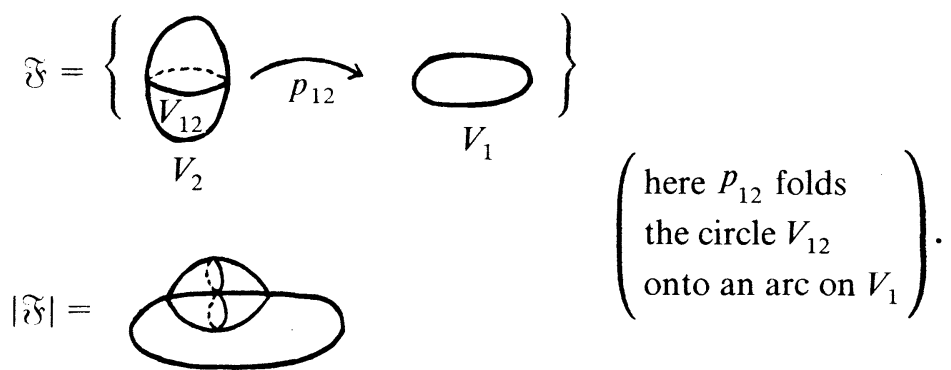

If $\mathfrak{F} \in \mathscr{A}_{U}$ then $|\mathfrak{F}|$ is P.L. homeomorphic to a real algebraic set whose singular stratification coincides with the smooth stratification of $|\mathfrak{F}|\left[\mathbf{A} \mathbf{K}_{\mathbf{4}}\right]$. (We say that two stratified sets are P.L. homeomorphic if they have smooth subdivisions which are isomorphic as stratified sets.) Note that an algebraic set has a canonical stratification up to P.L. homeomorphism since any two semialgebraic stratifications have a common subdivision. We know that any algebraic set is P.L. homeomorphic to $|\mathfrak{F}|$ for some $\mathfrak{F} \in \mathscr{A}$; in fact $\mathfrak{F} \in \mathscr{A}_{M U F}\left[\mathbf{A K}_{\mathbf{3}}\right]$.

We adopt the convention that if $\mathscr{S}$ is any subset of $\mathscr{T}$ or $\mathscr{A}$, then

$$
|\mathscr{S}|=\{|\mathfrak{F}| \mid \mathfrak{F} \in \mathscr{S}, \partial \mathfrak{F}=\varnothing\} .
$$


So up to P.L. homeomorphism, we have

$$
\{\text { Real algebraic sets }\}=\left|\mathscr{A}_{M U F}\right| .
$$

Let $\tau:|\mathscr{A}| \hookrightarrow|\mathscr{T}|$ be the forgetful map. In $\left[\mathbf{A K}_{2}\right]$ it is shown that for any $\mathfrak{F} \in \mathscr{T}$ there is a regular $\mathfrak{F}^{\prime} \in \mathscr{T}$ with $\left|\mathfrak{F}^{\prime}\right|=|\mathfrak{F}|$ and $\mathfrak{F}^{\prime}$ has the same type as $\mathfrak{F}$. In $\left[\mathbf{A K} \mathbf{K}_{4}\right]$ it is shown that $\tau$ maps $\left|\mathscr{A}_{S F}\right|$ onto $\left|\mathscr{T}_{R S F}\right|$. So up to P.L. homeomorphism, we have the following commutative diagram:

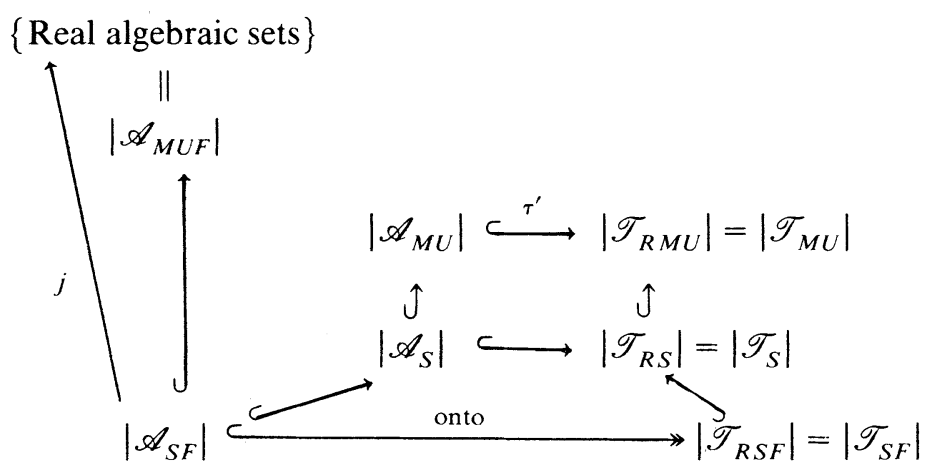

where the horizontal maps are restrictions of $\tau$, and $j$ is induced by composition. Hence proving $j$ (or less likely $\tau^{\prime}$ ) onto would give a complete topological characterization of real algebraic sets. Anyhow, the elements of $\left|\mathscr{T}_{R S}\right|$ give a good picture of what real algebraic sets look like. In this paper we give an obstruction theory to decide which stratified spaces lie in $\left|\mathscr{T}_{R S}\right|$. Many of the results of this paper remain valid when we replace $\mathscr{T}_{R S}$ with some other subsets of $\mathscr{T}$. For clarity we will stick with $\mathscr{T}_{R S}$ throughout the paper, and leave it to the reader to verify these possible variations of the results. For brevity we will denote $T R=\mathscr{T}_{R S}$ and $A R=\mathscr{A}_{S}$.

We are now ready to state our main result.

THEOREM 1.2. If $\mathscr{H} \in T R$ and $\mathscr{H}=\partial \mathfrak{F}^{\prime}$ for some seminormal $\mathfrak{F}^{\prime} \in T R$, then there exists $\mathfrak{F} \in T R$ such that

(i) $\partial \mathfrak{F}=\mathfrak{G}$,

(ii) $|\mathfrak{F}| \approx$ cone $|\mathfrak{S}|$.

Above $\approx$ means a stratified space isomorphism. This theorem is the obvious generalization of the classical spine theorem which allowed us to classify the real algebraic sets with isolated singularities $\left[\mathbf{A K}_{\mathbf{6}}\right]$. Notice in particular (ii) implies that if must be seminormal.

Theorem 1.2 is the basic result that allows us to define an obstruction theory of putting $T R$ structures on stratified spaces. Let $Y$ be a stratified space of dimension $n$ such that each connected smooth stratum has a trivial normal bundle. Let $Z$ be the $s$-skeleton of $Y$, i.e. $Z$ is the union of $i$-strata for all $i \leqslant s$. Suppose $Y-Z$ has a $T R$ structure respecting the trivializations, so there is a $\mathfrak{F} \in T R$ with $|\Im|=Y-Z$. Also by assumption if $\sigma$ is an open connected $s$-stratum then $\mathfrak{F}$ restricts to $\mathfrak{F}^{\prime} \times \sigma$ 


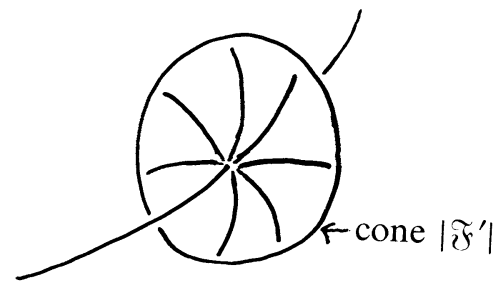

Figure 8

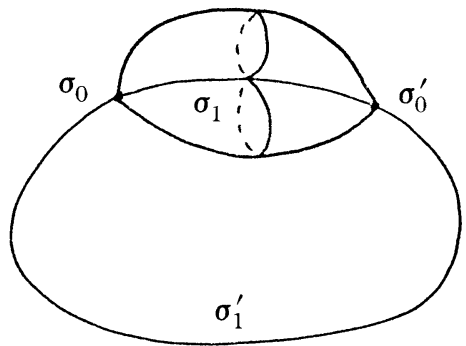

Figure 9

on the boundary of the tubular neighborhood of $\sigma$ for some $\mathfrak{F}^{\prime} \in T R$, in particular $\left|\mathfrak{F}^{\prime} \times \sigma\right|=\left|\mathfrak{F}^{\prime}\right| \times \sigma$. Let $Z^{\prime}=Z-\sigma$. If we can find $\mathfrak{F}^{\prime \prime} \in T R$ with $\partial \mathfrak{F}^{\prime \prime}=\mathfrak{F}^{\prime}$ and $\left|\mathfrak{F}^{\prime \prime}\right|=$ cone on $\left|\mathfrak{F}^{\prime}\right|$, then we can extend $\mathfrak{F}$ to $Y-Z^{\prime}$ across $\sigma$ by $\mathfrak{F} \cup_{\partial}\left(\mathfrak{F}^{\prime \prime} \times \sigma\right)$. See Figure 8.

Theorem 1.2 reduces the awkward question of finding such an $\widetilde{F}^{\prime \prime}$ to a bordism problem, namely it suffices to prove that $\mathfrak{F}^{\prime}$ bounds a seminormal resolution tower. In case $Z$ is a point, then the theorem roughly says that $\left|\mathfrak{F}^{\prime}\right|$ can occur as a link of a point in an algebraic variety if $\mathfrak{F}^{\prime}$ bounds.

As an example, let $Y$ be the stratified set in Figure 9. Let $Z_{i}$ be the $i$ th skeleton of $Y$. Then $Y-Z_{1}$ has a $T R$ structure since it is smooth. The link of $\sigma_{1}$ is four points; they bound two arcs. Let $\mathfrak{F}_{1}$ be the resolution tower

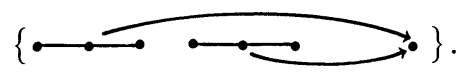

Then we can extend the $T R$ structure on $Y-Z_{1}$ by $\mathfrak{F}_{1} \times \sigma_{1}$ across $\sigma_{1}$, since

$$
\left|\mathfrak{\mho}_{1}\right|=\gamma \text {. }
$$

So we have a $T R$ structure on $Y-Z_{0}$ and $Z_{0}=\left\{\sigma_{0}, \sigma_{0}^{\prime}\right\}$. This induces $T R$ structures on the links of $\sigma_{0}$ and $\sigma_{0}^{\prime}$ by

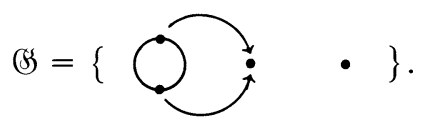

Let

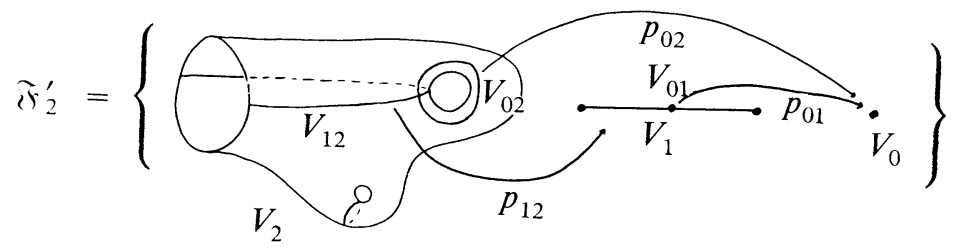

where $p_{12}$ is the folding map, $p_{01}$ and $p_{02}$ are collapsing maps. Then $\partial \mathfrak{F}_{2}^{\prime}=(\mathfrak{S}$ and $\mathfrak{F}_{2}^{\prime}$ is seminormal. Theorem 1.2 tells us that there is an $\mathfrak{F}_{2} \in T R$ such that $\partial \mathfrak{F}_{2}=(\mathfrak{S}$ 
and $\left|\mathfrak{F}_{2}\right| \approx$ cone $|\mathscr{G}|$, so we can extend the $T R$ structure across $\sigma_{0}$ and $\sigma_{0}^{\prime}$ to all of $Y$ by adding two copies of $\mathfrak{F}_{2}$. For example, we can take

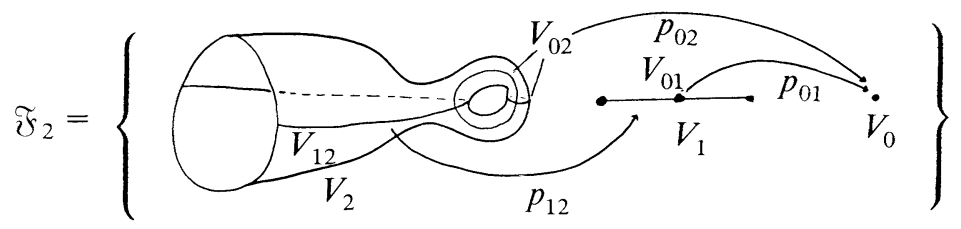

since

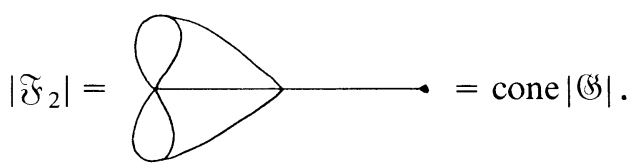

In higher dimensions, it is often harder to find $\mathfrak{F}_{2}$ than $\mathfrak{F}_{2}^{\prime}$, which is any seminormal bordism with $\partial \mathfrak{F}_{2}^{\prime}=\mathfrak{G}$, but Theorem 1.2 allows us to find such $\mathfrak{F}_{2}$ from $\mathfrak{F}_{2}^{\prime}$.

2. Properties of resolution towers. It is shown in $\left[\mathbf{A K}_{2}\right]$ that if $\mathfrak{F} \in \mathscr{T}_{M U}$, then there exists $\mathfrak{F}^{\prime} \in \mathscr{T}_{R M U}$ with $\left|\mathfrak{F}^{\prime}\right|=|\mathfrak{F}|$. Here we study some further topological properties of resolution towers. Our main goal is to be able to modify resolution towers to nicer ones as in the above result.

Proposition 2.1. Let $\mathfrak{F} \in T R$ be seminormal with $V_{0}(\mathfrak{F})=a$ point. Let $k$ be the smallest integer $\geqslant 1$ such that $V_{k}(\mathfrak{F})$ has closed manifold components (if it exists). Then there exists a seminormal $\mathfrak{F}^{\prime} \in T R$ such that

(1) $\partial \mathfrak{F}^{\prime}=\partial \mathfrak{F}$

(2) $\mathfrak{F}_{k-1}^{\prime}=\mathfrak{\mho}_{k-1}$,

(3) $V_{i}\left(\mathfrak{F}^{\prime}\right)=$ the union of connected components of $V_{i}(\mathfrak{F})$ with nonempty boundary for $i \geqslant k$.

(4) $\left|\mathfrak{A}_{i}\left(\mathfrak{F}^{\prime}\right)\right|=\left|\mathfrak{A}_{i}(\mathfrak{F})\right| \cap V_{i}\left(\mathfrak{F}^{\prime}\right)$, for $i \geqslant k$.

Proof. Let $\mathfrak{F}=\left\{V_{i}, \mathfrak{A}_{i}, p_{i}\right\}_{i=0}^{n}$. Define $\mathfrak{F}_{k-1}^{\prime}=\mathfrak{F}_{k-1}$ and for each $i \geqslant k$ let $V_{i}^{\prime}$ be the union of connected components of $V_{i}$ which have nonempty boundary. For all $1 \leqslant j<i$ let $V_{j i}^{\prime}=p_{j i}^{-1}\left(V_{j}^{\prime}\right) \cap V_{i}^{\prime}$, and $p_{j i}^{\prime}: V_{j i}^{\prime} \rightarrow V_{j}^{\prime}$ be the restriction of $p_{j i}$. Let $V_{0 i}^{\prime}=V_{i}^{\prime} \cap \bigcup_{j=1}^{i-1}\left(V_{j i}-V_{j i}^{\prime}\right)$, and $p_{0 i}^{\prime}: V_{0 i}^{\prime} \rightarrow V_{0}^{\prime}$ be the constant map. Let $\mathfrak{A}_{j i}^{\prime}$ be the sheets of $V_{j i}^{\prime}$ and $\mathfrak{U}_{i}^{\prime}=\bigcup_{j=0}^{i-1} \mathfrak{U}_{j i}^{\prime}$ and $p_{i}^{\prime}=\left\{p_{0 i}^{\prime}, \ldots, p_{i-1, i}^{\prime}\right\}$. Then $\mathfrak{F}^{\prime}=$ $\left\{V_{i}^{\prime}, \mathfrak{A}_{i}^{\prime}, p_{i}^{\prime}\right\}_{i=0}^{n}$ gives the required resolution tower.

Definition. Let $\mathfrak{F}, \mathfrak{F}^{\prime} \in T R$ with height $\left(\mathfrak{F}^{\prime}\right) \leqslant \operatorname{height}(\mathfrak{F})$. Then a transverse tower map $\phi: \mathfrak{F}^{\prime} \rightarrow \mathfrak{F}$ is a collection of maps $\phi_{i}: V_{i}\left(\mathfrak{F}^{\prime}\right) \rightarrow V_{i}(\mathfrak{F})$ for $i=$ $0,1, \ldots$, height $\left(\mathfrak{F}^{\prime}\right)$ which are tico-transverse to $\mathfrak{A}_{i}(\mathfrak{F})$ such that for all $0 \leqslant j<i \leqslant$ height $\left(\mathfrak{F}^{\prime}\right)$

(a) $\mathfrak{A}_{j i}\left(\mathfrak{F}^{\prime}\right)=\phi_{i}^{*} \mathfrak{A}_{j i}(\mathfrak{F})$, 
(b) The following commutes:

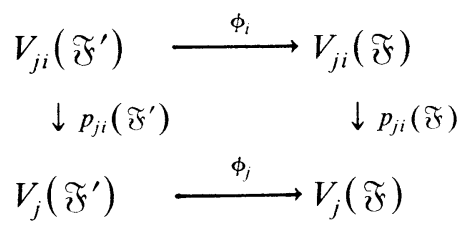

where $V_{j i}\left(\mathfrak{F}^{\prime}\right)=\left|\mathfrak{A}_{j i}\left(\mathfrak{F}^{\prime}\right)\right|$.

We say $\mathfrak{F}^{\prime}$ is a subtower of $\mathfrak{F}$ if all $\phi_{i}$ are inclusions. We call a subtower $\mathfrak{F}^{\prime}$ a codimension $k$ subtower if all $V_{i}\left(\mathfrak{F}^{\prime}\right)$ are codimension $k$ submanifolds of $V_{i}(\mathfrak{F})$.

Definition. Let $\mathfrak{F} \in T R$ with $n=\operatorname{height}(\mathfrak{F})$. Then $\mathscr{A}=\left\{A_{j}\right\}_{j=k}^{m}$ where $k \leqslant m$ $\leqslant n$ is called a compatible family of submanifolds of $\mathfrak{F}$ if each $A_{j}$ is a compact smooth submanifold of $V_{j}(\mathfrak{F})$ (it can be empty) such that

(1) $A_{j}$ is transverse to $\mathfrak{A}_{j}(\mathfrak{F})$, for all $j \geqslant k$.

(2) $p_{j i}(\mathfrak{F})$ is tico-transverse to $A_{j}$, for all $i>j \geqslant k$.

(3) $p_{j i}(\mathfrak{F})^{-1}\left(A_{j}\right)=V_{j i}(\mathfrak{F}) \cap A_{i}, p_{j i}(\mathfrak{F})^{-1}\left(\partial A_{j}\right)=V_{j i}(\mathfrak{F}) \cap \partial A_{i}$, for $k \leqslant j<i \leqslant$ $m$. More generally we define $\mathscr{A}=\left\{\mathscr{B}_{i}\right\}_{i=k}^{m}$ to be a compatible family of tims in $\mathfrak{F}$ if each $\mathscr{B}_{i}=\left\{A_{j i}\right\}_{j \in \Lambda}$ is a tim in $V_{i}(\mathfrak{F})$ such that each $\mathscr{A}_{\alpha}=\left\{A_{\alpha i}\right\}_{i=k}^{m}$ is a compatible family of submanifolds of $\mathfrak{F}$ for all $\alpha \subset \Lambda$ where $A_{\alpha i}=\bigcap_{j \in \alpha} A_{j i}$. In particular, compatible family of ticos is defined. Also we define $\left.\mathscr{A}\right|_{\partial \Im}$ to be the compatible family of tims $\left\{\left.\mathscr{B}_{i}\right|_{\partial \mathfrak{r}}\right\}$.

Lemma 2.2. Let $\mathfrak{F} \in T R, m \leqslant$ height( $\mathfrak{F})$, and let $\mathscr{A}=\left\{A_{i}\right\}_{i=k}^{m-1}$ be a compatible family of submanifolds in $\mathfrak{F}$. Then there exists a smooth submanifold $A_{m}$ in $V_{m}(\mathfrak{F})$ such that $\mathscr{A}^{\prime}=\left\{A_{i}\right\}_{i=k}^{m}$ is a compatible family of submanifolds in $\mathfrak{F}$.

Proof. Let $\mathscr{B}_{j m}=p_{j m}(\mathfrak{F})^{*}\left\{A_{j}\right\}$ and $\mathscr{B}_{m}=\bigcup_{j=k}^{m-1} \mathscr{B}_{j m}$. Then we claim that $\mathscr{B}_{m}$ is a subtim in $\bigcup_{j \geqslant k} \mathfrak{A}_{j m}(\mathfrak{F})$. Given this, we can take a normal thickening $A_{m}$ of $\mathscr{B}_{m}$ in $V_{k}(\mathfrak{F})$. So by construction $\mathscr{A}^{\prime}$ satisfies the conditions (1) and (3) of a compatible family. To check (2) take any connected component $S_{0}$ of a stratum of $V_{i}$ with $S_{0} \subset V_{m i}$. We must show that $p_{m i}:: S_{0} \rightarrow V_{m}$ is transverse to $A_{m}$. Let $S_{1}$ be the stratum of $V_{m}$ with $S_{1} \supset p_{m i}\left(S_{0}\right)$. Suppose first that $S_{0} \subset V_{j i}$ for some $j<m$. Then $S_{1} \subset\left|\mathfrak{A}_{m}(\mathfrak{F})\right|$ so since $p_{j i} \mid: S_{0} \rightarrow V_{j}$ is transverse to $A_{j},\left.p_{j i}\right|_{S_{0}}=\left.p_{j m} \circ p_{m i}\right|_{S_{0}}$ and $S_{1} \cap A_{m}=S_{1} \cap p_{j m}^{-1}\left(A_{j}\right)$ we know that $\left.p_{m i}\right|_{S_{0}}$ must be transverse to $A_{m}$. Now suppose that $S_{0} \subset V_{m i}-\bigcup_{j<m} V_{j i}$. Because $\mathfrak{F}$ has type $U$ we are done since $p_{m i}$ submerses $S_{0}$ to $V_{m}-\left|\mathfrak{A}_{m}\right|$. Now it remains to show that $\mathscr{B}_{m}$ is a subtim in $\bigcup_{j \geqslant k} \mathfrak{A}_{j m}(\mathfrak{F})$, but this follows from Lemma 1.1. See Figure 10.

Lemma 2.3. Let $\mathfrak{F} \in T R, m \leqslant$ height( $\mathfrak{F})$, and let $\mathscr{A}=\left\{\mathscr{A}_{i}\right\}_{i=k}^{m-1}$ and $\mathscr{B}=\left\{\mathscr{B}_{i}\right\}_{i=k}^{m}$ be compatible families of tims in $\mathfrak{F}$ with $\mathscr{B}_{i} \subset \mathscr{A}_{i}$ for all $i<m$. Then there exists a tim $\mathscr{A}_{m}$ in $V_{m}(\mathfrak{F})$ such that $\mathscr{B}_{m} \subset \mathscr{A}_{m}$ and $\mathscr{A}^{\prime}=\left\{\mathscr{A}_{i}\right\}_{i=k}^{m}$ is a compatible family of tims. Furthermore, if there is a tim $\mathscr{C}_{m}$ in $\partial V_{m}(\mathfrak{F})$ such that $\left.\mathscr{A}\right|_{\partial \mathfrak{F}_{5}} \cup\left\{\mathscr{C}_{m}\right\}$ is a compatible family of tims in $\partial \mathfrak{F}$, then we can choose $\mathscr{A}_{m}$ such that $\left.\mathscr{A}_{m}\right|_{\partial \mathfrak{F}}=\mathscr{C}_{m}$. 


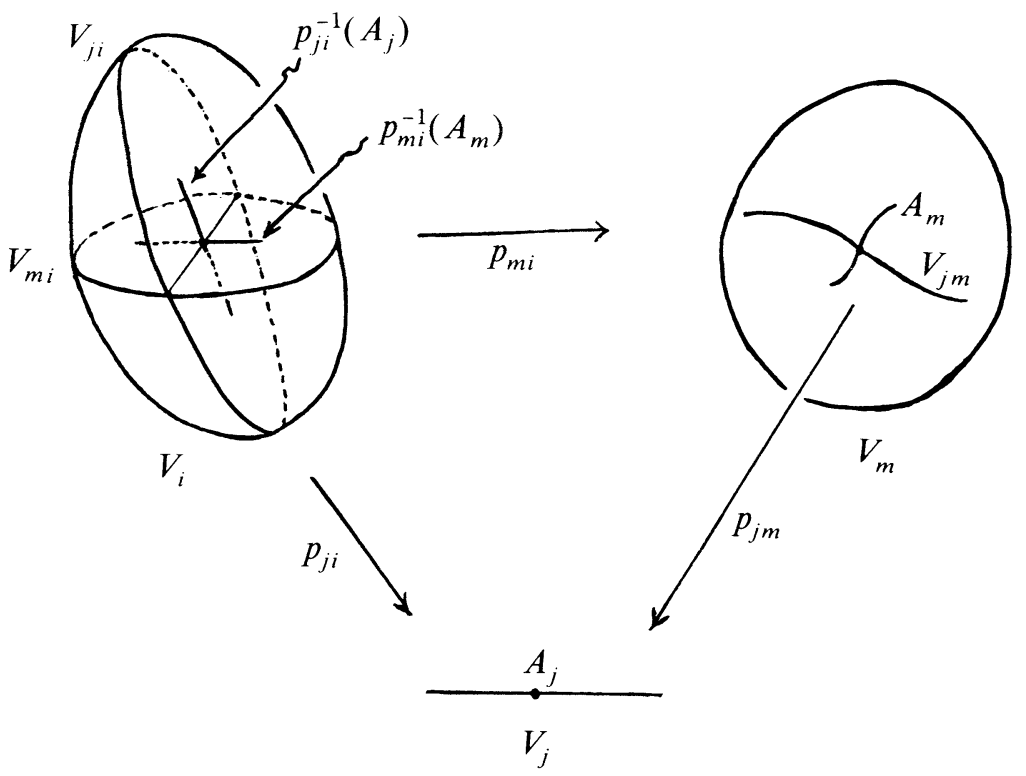

Figure 10

Proof. The proof follows by applying Lemma 2.2 to the sheets of $\mathscr{A}$. To get the relative version, recall that the sheets of $\mathscr{A}_{m}$ are obtained by taking normal thickenings and there is relative existence for normal thickenings.

LeMma 2.4. Let $\mathfrak{F} \in T R, m \leqslant \operatorname{height(} \mathfrak{F})$. Let $\mathscr{A}=\left\{\mathscr{A}_{i}\right\}_{i=k}^{m-1}$ be a compatible family of tims in $\mathfrak{F}$ and let $\mathscr{B}=\left\{\mathscr{B}_{i}\right\}_{i=k}^{m}$ be a compatible family of ticos in $\mathfrak{F}$. Suppose $\mathscr{B}_{i} \subset \mathscr{A}_{i}$ for $k \leqslant i \leqslant m-1$ and each sheet of each $\mathscr{A}_{i}-\mathscr{B}_{i}$ has codimension zero. Then there exists a tim $\mathscr{A}_{m}$ in $V_{m}(\mathfrak{F})$ so that $\mathscr{B}_{m} \subset \mathscr{A}_{m}$, each sheet of $\mathscr{A}_{m}-\mathscr{B}_{m}$ has codimension zero, $\mathscr{A}^{\prime}=\left\{\mathscr{A}_{i}\right\}_{i=k}^{m}$ is a compatible family of tims and $\mathscr{B}^{\prime}=\left\{\mathscr{B}_{i}^{\prime}\right\}_{i=k}^{m}$ is a compatible family of ticos where $\mathscr{B}_{i}^{\prime}=\mathscr{B}_{i} \cup\left\{\partial_{+} A \mid A \in \mathscr{A}_{i}-\mathscr{B}_{i}\right\}$.

Proof. Apply Lemma 2.3 to obtain $\mathscr{A}_{m}$. The compatibility of the family $\left\{\mathscr{B}_{i}^{\prime}\right\}_{i=k}^{m}$ then follows easily from compatibility of $\left\{\mathscr{A}_{i}\right\}_{i=k}^{m}$ and $\left\{\mathscr{B}_{i}\right\}_{i=k}^{m}$.

Let $\mathfrak{F}, \mathfrak{F}^{\prime}, \mathfrak{F}^{\prime \prime} \in T R, \operatorname{dim}\left(\mathfrak{F}^{\prime}\right)=\operatorname{dim}\left(\mathfrak{F}^{\prime \prime}\right)$, such that $\partial \mathfrak{F}^{\prime}=\mathbb{H} \cup_{\jmath} \mathfrak{H}^{\prime}$ and $\partial \mathfrak{F}^{\prime \prime}$

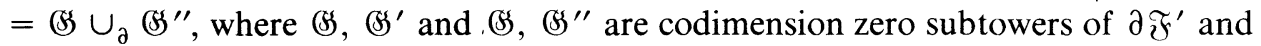
$\partial \mathfrak{F}^{\prime \prime}$, respectively, with $\partial \mathfrak{G}=\partial \mathscr{S}^{\prime}=\partial \mathscr{F}^{\prime \prime}$, and $\mathfrak{F}=\mathfrak{F}^{\prime} \cup \mathfrak{F}^{\prime \prime}$ obtained by gluing along Ss. See Figure 11.

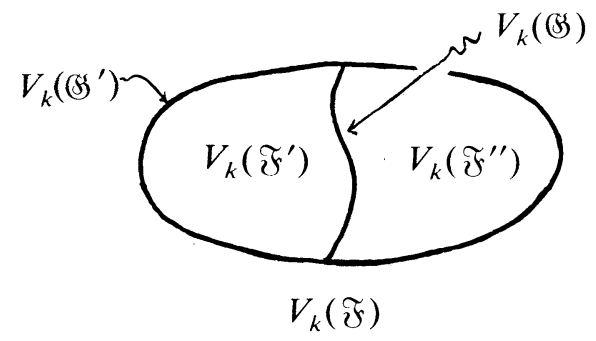

Figure 11 
Now given these notations we have the following proposition.

Proposition 2.5. Let $A_{k}$ be a compact codimension zero submanifold of $V_{k}\left(\mathfrak{F}^{\prime \prime}\right) \cap$ int $V_{k}(\mathfrak{F})$ such that $A_{k} \cap\left|\mathscr{U}_{k}(\mathfrak{F})\right|=\varnothing$. Let $\mathscr{B}^{*}=\left\{\mathscr{B}_{i}^{*}\right\}_{i \geqslant k}$ be a compatible family of ticos in $\mathfrak{F}^{\prime}$ and let $\mathscr{B}_{k}$ be a tico in $V_{k}\left(\mathfrak{F}^{\prime}\right) \cup A_{k}$ transverse to $V_{k}(\mathfrak{B S})$ and $\mathfrak{A}_{k}(\mathfrak{B S})$ such that $\mathscr{B}_{k} \cap V_{k}\left(\mathfrak{F}^{\prime}\right)=\mathscr{B}_{k}^{*} \cup \mathscr{B}_{k}^{* *}$ and $\mathscr{B}_{k}^{* *}$ is a separating tico. Then after a small isotopy of $A_{k}$ and $\mathscr{B}_{k}^{* *}$ we can find a compatible family of codimension zero submanifolds $\mathscr{A}=\left\{A_{i}\right\}_{i \geqslant k}$ in $\mathfrak{F}^{\prime \prime}$, and ticos $\mathscr{B}_{i}$ in $V_{i}\left(\mathfrak{F}^{\prime}\right) \cup A_{i}$ for all $i>k$, and $\overline{\widetilde{F}} \in T R$ such that

(a) $\bar{\mho}_{k-1}=\widetilde{\mho}_{k-1}^{\prime}$,

(b) $V_{i}(\overline{\mathfrak{F}})=V_{i}\left(\mathfrak{F}^{\prime}\right) \cup A_{i}$ for $i \geqslant k$

(c) $\mathfrak{A}_{i}(\overline{\mathfrak{F}})=\mathfrak{A}_{i}(\mathfrak{F}) \cap V_{i}(\overline{\mathfrak{F}}), p_{i}^{\prime}(\overline{\mathfrak{F}})=\left.p_{i}(\mathfrak{F})\right|_{\mathfrak{A}_{i}(\overline{\mathfrak{F}})}$,

(d) $\mathscr{B}=\left\{\mathscr{B}_{i}\right\}_{i \geqslant k}$ is a compatible family of ticos in $₹$.

(e) $\mathscr{B}_{i}^{*} \subset \mathscr{B}_{i} \cap V_{i}\left(\widetilde{F}^{\prime}\right)$ for all $i \geqslant k$.

Furthermore, if $\mathscr{B}_{k}^{* *}=\left\{\partial_{+} D \mid D \in \mathscr{D}_{k}\right\}$ for some tim $\mathscr{D}_{k}$ with $\left|\mathscr{D}_{k}\right| \cap \partial V_{k}(\mathfrak{S})=\varnothing$, then we may also conclude that $\left|\mathscr{B}_{i}\right| \cap \partial V_{i}(\mathfrak{G S})=\left|\mathscr{B}_{i}{ }^{*}\right| \cap \partial V_{i}(\mathfrak{G S})$ for all $i \geqslant k$. See Figure 12.

Proof. After a small isotopy of $A_{k}$ and $\mathscr{B}_{k}^{* *}$ we can assume that all $p_{k i}(\mathfrak{F})$ 's are tico-transverse to $A_{k}$ and $\mathscr{B}_{k}^{* *}$ for all $i>k$. Let $\mathscr{D}_{k}$ be a codimension zero tim in $V_{k}\left(\mathfrak{F}^{\prime}\right)$ so that $\left\{\partial_{+} D_{1} \mid D \in \mathscr{D}_{k}\right\}=\mathscr{B}_{k}^{* *}$. By Lemma 2.4 with $m=k+1$ and $\mathscr{A}_{k}=\mathscr{D}_{k} \cup \mathscr{B}_{k}^{*}$, there is a tim $\mathscr{A}_{k+1}=\mathscr{D}_{k+1} \cup \mathscr{B}_{k+1}^{*}$ so that $\left\{\mathscr{A}_{k}, \mathscr{A}_{k+1}\right\}$ is a compatible family. In case $\left|\mathscr{D}_{k}\right| \cap \partial V_{k}($ (B) $)=\varnothing$ we first use Lemma 2.4 to lift $\left.\mathscr{A}_{k}\right|_{\text {G }}$ to a tim $\left.\mathscr{D}_{k+1}^{*} \cup \mathscr{B}_{k+1}^{*}\right|_{\mathscr{G}}$ in $V_{k+1}(\mathbb{G S})$ so that $\left|\mathscr{D}_{k+1}^{*}\right| \cap \partial V_{k+1}(\mathbb{S})=\varnothing$, then lift $\left.\mathscr{A}_{k}\right|_{\mathscr{S}^{\prime}}$ to a tim $\left.\mathscr{D}_{k+1}^{\prime} \cup \mathscr{B}_{k+1}^{*}\right|_{\mathscr{S}^{\prime}}$ in $V_{k+1}\left(\mathscr{S}^{\prime}\right)$ so that $\left|\mathscr{D}_{k+1}^{\prime}\right| \cap \partial V_{k+1}(\mathbb{G})=\varnothing$. Taking unions of sheets in $\mathscr{D}_{k+1}^{\prime}$ and in $\mathscr{D}_{k+1}^{\prime}$ we get a tim $\mathscr{D}_{k+1}^{\prime \prime}$ in $\partial V_{k+1}\left(\mathfrak{F}^{\prime}\right)$ so that $\left.\mathscr{D}_{k+1}^{\prime \prime} \cup \mathscr{B}_{k+1}^{*}\right|_{\partial \widetilde{Y}^{\prime}}$ lifts $\left.\mathscr{A}_{k}\right|_{\partial \widetilde{Y}^{\prime}}$ and $\left|\mathscr{D}_{k+1}^{\prime \prime}\right| \cap \partial V_{k+1}(\mathscr{S})=\varnothing$. We then pick our above lifting $\mathscr{D}_{k+1}$ so that $\left.\mathscr{D}_{k+1}\right|_{\partial \widetilde{\mho}^{\prime}}=\mathscr{D}_{k+1}^{\prime \prime}$, in particular $\left|\mathscr{D}_{k+1}\right| \cap \partial V_{k+1}(\mathbb{B S})=\varnothing$. Continuing in this manner we obtain a compatible family of tims $\left\{\mathscr{A}_{i}\right\}_{i \geqslant k}$ in $\mathfrak{F}^{\prime}$ where $\mathscr{A}_{i}=\mathscr{D}_{i} \cup \mathscr{B}_{i}^{*}, i \geqslant k$, and $\left\{\mathscr{D}_{i}\right\}_{i \geqslant k}$ is a compatible family of tims in $\mathfrak{F}^{\prime}$ and $\left|\mathscr{D}_{i}\right| \cap \partial V_{i}(\mathscr{G})=\varnothing$. So we have a compatible family of ticos in $\mathscr{F}^{\prime}, \mathscr{B}^{\prime}=\left\{\mathscr{B}_{i}^{\prime}\right\}_{i \geqslant k}$ where $\mathscr{B}_{i}^{\prime}=\mathscr{B}_{i}^{*} \cup\left\{\partial_{+} D \mid D \in \mathscr{D}_{i}\right\}$. Notice $\left|\mathscr{B}_{i}^{\prime}\right| \cap \partial V_{i}\left((\mathfrak{S})=\left|\mathscr{B}_{i}^{*}\right| \cap \partial V_{i}(\right.$ (S) $)$ for all $i \geqslant k$.

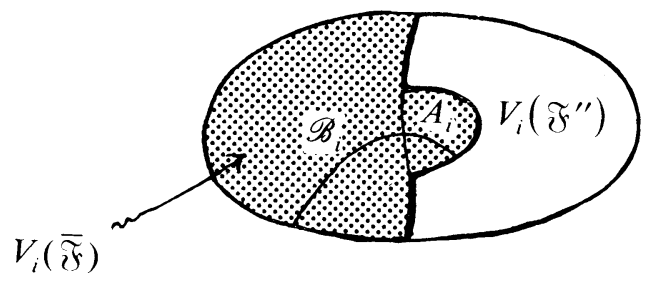

$V_{i}(\tilde{i})$ 
Form a tim $\mathscr{B}_{k}^{\prime \prime}$ in $\mathfrak{F}^{\prime \prime}$ with $\mathscr{B}_{k}^{\prime \prime} \cap A_{k}=\mathscr{B}_{k} \cap A_{k}$, and $\mathscr{B}_{k}^{\prime \prime} \cap V_{k}(\mathfrak{S})=\mathscr{B}_{k} \cap$ $V_{k}(\mathscr{G})$ by taking $\mathscr{B}_{k} \cap\left(A_{k} \cup V_{k}(\mathscr{G})\right)$ and adding a collar along $\mathscr{B}_{k} \cap\left(\partial_{+} A_{k} \cup\right.$ $\left.V_{k}(\mathfrak{S})-\partial_{-}\left(A_{k}\right)\right)$. By repeated applications of Lemma 2.4 we obtain a compatible family of tims $\left\{\mathscr{B}_{i}^{\prime \prime}\right\}_{i \geqslant k}$ in $\mathfrak{F}^{\prime \prime}$ with $\mathscr{B}_{i}^{\prime \prime} \cap V_{i}(\mathscr{H})=\mathscr{B}_{i}^{\prime} \cap V_{i}(\mathscr{H})$. Now apply Lemma 2.2 repeatedly to $A_{k}$ to obtain a compatible family of submanifolds $\left\{A_{i}\right\}_{i \geqslant k}$ in $\mathfrak{F}^{\prime \prime}$ with each $A_{i}$ in general position with $\mathfrak{A}_{i}\left(\mathfrak{F}^{\prime \prime}\right)$ and $\mathscr{B}_{i}^{\prime \prime}$. Furthermore, an examination of the proof of Lemma 2.2 reveals that we may guarantee that each $A_{i} \cap \mathscr{B}_{i}^{\prime \prime}$ is a tico in $A_{i}$. (Do this by making your thickenings very thin. Actually the proof of the normal thickening lemma will do this automatically.) Then $\overline{\mathscr{A}}=\left\{\bar{V}_{i}\right\}_{i \geqslant 0}$, where

$$
\bar{V}_{i}= \begin{cases}V_{i}\left(\mathfrak{F}^{\prime}\right) & \text { for } i<k, \\ V_{i}\left(\mathfrak{F}^{\prime}\right) \cup A_{i} & \text { for } i \geqslant k,\end{cases}
$$

is a compatible family of codimension zero submanifolds in $\mathfrak{F}$; hence it defines a subtower $\overline{\mathfrak{F}}=\left\{\bar{V}_{i}, \overline{\mathfrak{A}}_{i}, \bar{p}_{i}\right\}$ where $\overline{\mathfrak{A}}_{i}=\mathfrak{U}_{i}(\mathfrak{F}) \cap \bar{V}_{i}$. Then $\mathscr{B}=\left\{\mathscr{B}_{i}\right\}_{i \geqslant k}$ where $\mathscr{B}_{i}$ $=\left|\mathscr{B}_{i}^{\prime}\right| \cup{ }_{a}\left|\mathscr{B}_{i}^{\prime \prime} \cap A_{i}\right|$ is a compatible family of ticos in $\overline{\mathfrak{F}}$. See Figure 13.

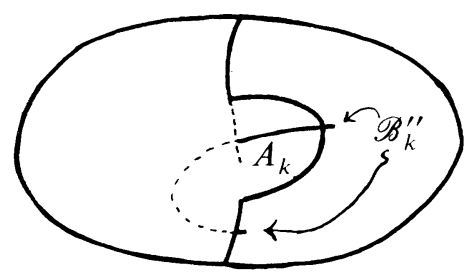

Figure 13

Corollary 2.6. Under the same hypothesis of Proposition 2.5 and $\left|\mathscr{B}_{i}{ }^{*}\right| \cap \partial V_{i}(\mathrm{GS})$ $=\varnothing$ for all $i \geqslant k,\left|\mathscr{D}_{k}\right| \cap \partial V_{k}(\mathbb{G S})=\varnothing$ where $\mathscr{B}_{k}^{* *}=\partial_{+} \mathscr{D}_{k}$, and $V_{0}(\mathfrak{G S})=$ a point, then there exists $\overline{\text { बS }} \in T R$ such that

(a) $\partial \overline{\mathbb{B S}}=\partial$ (S),

(b) $\overline{\mathscr{S}}_{k-1}=\mathbb{B S}_{k-1}$,

(c) $V_{k}(\overline{\text { (S) }})=\left(V_{k}(\right.$ (B) $\left.)-\operatorname{int}\left(\partial_{-} A_{k}\right)\right) \cup \partial_{+} A_{k}$,

(d) $\mathfrak{A}_{j k}(\overline{G S})=\mathfrak{H}_{j k}($ (S) for $0<j<k$,

(e) $\mathfrak{A}_{0 k}(\overline{\mathbb{S}})=\mathfrak{A}_{0 k}(\mathfrak{S}) \cup \partial \mathscr{B}_{k}$,

(f) $p_{j k}(\overline{\mathbb{G}})=p_{j k}($ (B) $)$ for $0<j<k, p_{0 k}(\overline{G S})=$ trivial map.

Proof. Let $\mathscr{G}^{*}=\partial \overline{\mathfrak{F}}-\operatorname{int}\left(\mathscr{S}^{\prime}\right)$, then $\mathscr{B S}^{*}$ satisfies $(\mathrm{a})$ and $(\mathrm{b})$ and $\mathfrak{U}_{i}\left(\mathbb{G}^{*}\right)=$ $\mathfrak{A}_{i}(\mathfrak{F}) \cap V_{i}\left(\mathbb{S}^{*}\right)$ and $p_{i}\left(\mathfrak{S}^{*}\right)=\left.p_{i}(\mathfrak{F})\right|_{\mathfrak{A}_{i}(\mathbb{B} *)^{*}}$. Then simply define $\overline{\mathscr{S}}$ by $\overline{\mathscr{G S}}_{k-1}=$

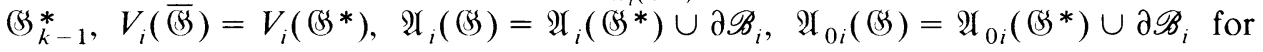
all $i \geqslant k, p_{j i}((B))=p_{j i}\left(B^{*}\right)$ for $0<j<i$, and $p_{0 i}((B))=$ trivial map. Notice since $A_{k}$ is disjoint from $\mathfrak{U}_{k}(\mathfrak{F}), \mathfrak{U}_{k}\left(\mathfrak{S H}^{*}\right)=\mathfrak{A}_{k}(\mathfrak{B S})$ so (c), (d), (e) are satisfied. Clearly (b) is satisfied and (a) is satisfied since $\left|\partial \mathscr{B}_{i}\right| \cap \partial V_{i}(\overline{G S})=\left|\mathscr{B}_{i}\right| \cap \partial V_{i}($ (S) $)=\varnothing$ by Proposition 2.5. 
Let $T^{m}$ be the $m$-torus; there is a basic tico in $T^{m}$ which we will denote by $\mathscr{T}^{m}=\left\{T_{i}\right\}_{i=1}^{m}$ with each $T_{i}=S^{1} \times \cdots \times S^{1} \times 0 \times S^{1} \times \cdots \times S^{1}$ where 0 lies in the $i$ th slot. Let $V^{m}$ be a smooth $m$-manifold; then $\mathscr{T}^{m}$ induces a tico in $V^{m} \# T^{m}$ which we will continue to denote by $\mathscr{T}^{m}$.

Proposition 2.7. Let $\mathbb{B S} \in T R$ be seminormal with $V_{0}(\mathbb{S S})=$ a point. Let $k$ be any integer $\leqslant$ height(BS) such that all components of $V_{i}$ (BS) have nonempty boundaries for $0<i \leqslant k$. Then there exists (S)' $\in$ TR such that

(a) $\partial \mathscr{S H}^{\prime}=\partial \mathrm{GS}$,

(b) $\mathfrak{S G}_{k-1}^{\prime}=\mathfrak{S G}_{k-1}$,

(c) $V_{k}\left(\mathfrak{S S}^{\prime}\right)=V_{k}(\mathfrak{G H}) \# T^{m}$, where $m=\operatorname{dim} V_{k}(\mathbb{B S})$, and the connected sum is taken along any disc we choose in $V_{k}(\mathfrak{B S})-\left|\mathfrak{A}_{k}(\mathfrak{G H})\right|$,

(d) $\mathfrak{A}_{k}\left(\mathfrak{S S}^{\prime}\right)=\mathfrak{A}_{k}($ (S) $) \cup \mathscr{T}^{m}, \mathfrak{A}_{0 k}\left(\mathfrak{S H}^{\prime}\right)=\mathfrak{A}_{0 k}($ (S) $) \cup \mathscr{T}^{m}$,

(e) (S' is seminormal.

PRoof. $T^{m-1}$ imbeds in $\mathbf{R}^{m+1}$ with trivial normal bundle (in fact it imbeds into $\mathbf{R}^{m}$ with trivial normal bundle, prove by induction). Hence

$$
T^{m-1} \times D^{2} \hookrightarrow \operatorname{int}\left(B^{m} \times[1,2]\right) \subset V_{k}((S)) \times[1,2],
$$

where $B^{m}$ is a ball in $V_{k}(\mathbb{S})$ in the complement of $\mathfrak{A}_{k}(\mathbb{B S})$. Let $C$ be a thickened arc connecting $B^{m} \times 1$ to $T^{m-1} \times \partial D^{2}=T^{m}$, so $C \approx B^{m} \times I$. Let $D^{\prime}$ be a smaller open disc in $D^{2}$, and $A_{k}=C \cup T^{m-1} \times\left(D-D^{\prime}\right)$, and let $\mathscr{B}_{k}$ be the tico corresponding to $\mathscr{T}^{m} \times I$ in $A_{k}$. Let $\mathfrak{F}=\mathfrak{S} \times[0,2], \mathfrak{F}^{\prime}=\mathfrak{G} \times[0,1], \mathfrak{F}^{\prime \prime}=\mathfrak{G S} \times$ $[1,2]$. See Figure 14. By choosing $C$ small we can make sure that $A_{k} \cap\left|\mathfrak{A}_{k}(\mathfrak{F})\right|=\varnothing$. By Corollary 2.6 we get $\overline{\text { (s) }}$ satisfying (a), (b) and

$$
\begin{aligned}
& V_{k}(\overline{\text { (S) }})=\left(V_{k}(\text { (S) }) \# T^{m}\right) \amalg T^{m} \quad \text { (Ш means disjoint union), } \\
& \mathfrak{A}_{k}(\overline{\mathbb{S}})=\left(\mathfrak{A}_{k}(\mathfrak{S}) \cup \mathscr{T}^{m}\right) \amalg \mathscr{T}^{m} \text {. } \\
& \mathfrak{A}_{0 k}(\mathfrak{g})=\left(\mathfrak{U}_{0 k}(\mathscr{G S}) \cup \mathscr{T}^{m}\right) \amalg \mathscr{T}^{m} \text {. }
\end{aligned}
$$

By construction $\overline{(S)}$ remains seminormal, so by Proposition 2.1 there exists seminormal SS' $^{\prime} \in T R$ satisfying the required properties.

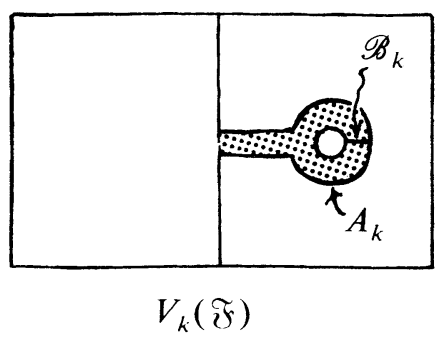

FIGURE 14 
3. Construction of spines. The following is a generalization of Proposition 4.2 of $\left[\mathbf{A K}_{1}\right]$.

Proposition 3.1. Let $V^{m}$ be an m-dimensional compact smooth manifold such that every component of $V$ has nonempty boundary. Let $\mathfrak{A}$ be a closed tico in $V$ such that for any component $W$ of $V, \chi(W-|\mathfrak{A}|) \equiv 0(\bmod 2)$, where $\chi$ denotes the Euler characteristic. Then there exists a compact codimension zero submanifold $A$ of $\operatorname{int}(V) \times$ $[1,2)$ and a tico $\mathscr{B}$ in $V \times[0,1] \cup A$ such that $: \partial \mathscr{B}=\tilde{\mathfrak{A}} \cup \mathscr{C}$ with $|\tilde{\mathfrak{A}}|=|\mathfrak{A}|$ and $\mathscr{C}$ is a closed tico in $\bar{V}=\left(V \times 1-\operatorname{int}\left(\partial_{-} A\right)\right) \cup \partial_{+}(A)$, and $\mathscr{C}$ is a spine of $\bar{V}$, that is, $\bar{V} /|\mathscr{C}| \approx \operatorname{cone}(\partial \bar{V})$ (notice $\partial \bar{V}=\partial V)$. Furthermore, $|\mathscr{B}| \cap V \times[0,1]=|\mathfrak{A}| \times[0,1]$ $\cup\left|\mathscr{B}^{* *}\right|$ where $\mathscr{B}^{* *}$ is a separating tico in $V \times[0,1]$. In fact, $\mathscr{B}^{* *}=\left\{\partial_{+} D \mid D \in \mathscr{E}\right\}$ for some codimension zero tim $\mathscr{E}$ in $V \times(0,1]$ so that $\partial_{-} \mathscr{E} \subset \operatorname{int}(V) \times 1$. See Figure 15.

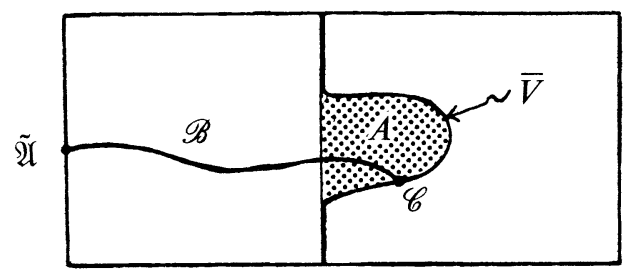

$V \times[0,2]$

FIGURE 15

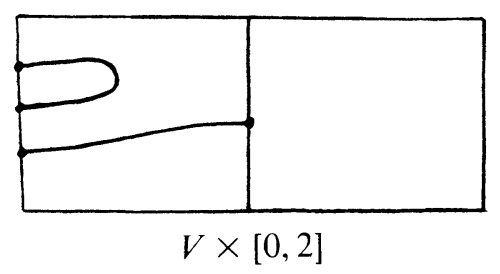

Figure 16

Proof. In case $m=1$ the proof is trivial. In this case $V$ is a disjoint union of closed intervals and $\mathfrak{A}$ has an odd number of points in each interval. We choose $\mathscr{A}$ and $\mathscr{B}$ in the obvious fashion as in Figure 16.

So we can assume $m \geqslant 2$. Also without loss of generality we will assume that $V$ is connected. Proving the proposition for each component would give the proof for all of $V$. We claim that we can pick a collection of balls $\mathscr{D}=\left\{D_{i}\right\}_{i=1}^{r}$ in interior $(V)$ such that (see Figure 17)

(a) $\cup_{i=1}^{r} D_{i} \cup|\mathfrak{A}|$ is a spine of $V$.

(b) $\left\{\partial D_{i}\right\}_{i=1}^{r} \cup \mathfrak{A}$ is a tico in $V$.

(c) $\cup D_{i}-\left(\bigcup \partial D_{i} \cup|\mathfrak{A}|\right)$ is a union of an even number of open balls $\bigcup_{j=1}^{s} B_{j}$, $s=2 p$.

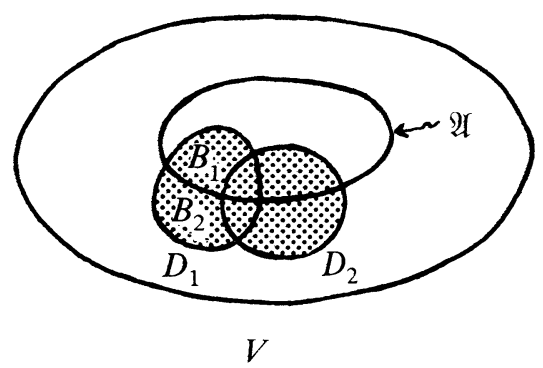

Figure 17 


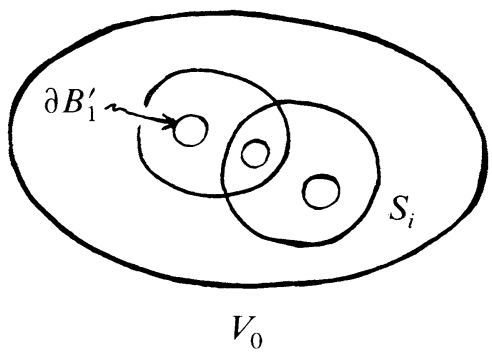

Figure 18

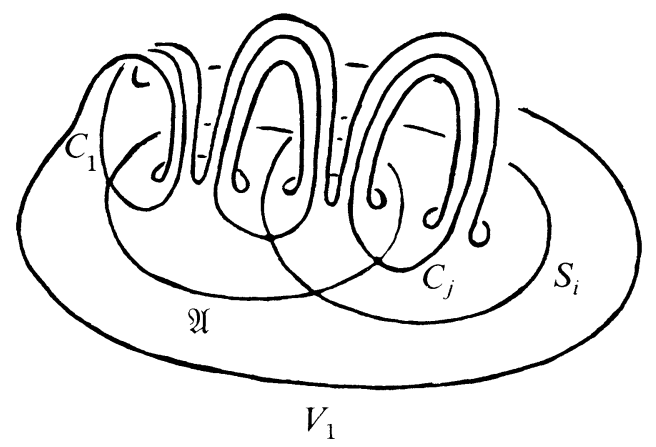

Figure 19

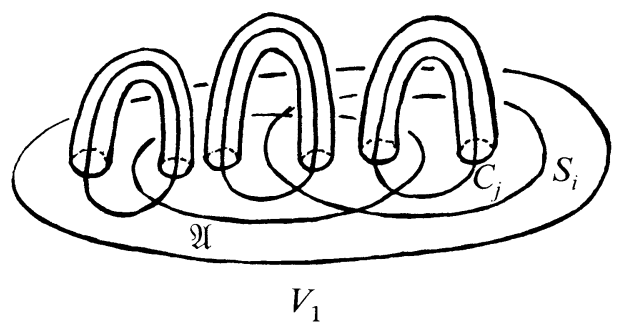

FIGURE 20

Clearly this can be done by covering the complement of an open collar of $\partial V$ by very small round balls. The only thing which is not clear is the evenness claim in (c). We see this as follows. Say $s$ is the number of open balls $\cup D_{i}-\left(\cup \partial D_{i} \cup|\mathfrak{A}|\right)$; then modulo 2 we have the equations

$$
\chi(V)=\chi\left(\bigcup_{i=1}^{r} D_{i} \cup|\mathfrak{A}|\right)=\chi\left(\bigcup_{i=1}^{r} \partial D_{i} \cup|\mathfrak{A}|\right)+s=\chi(|\mathfrak{A}|)+s .
$$

The last equality can be proven by induction on $r$. Hence $s=\chi(V-|\mathfrak{A}|)$ so $s$ must be an even integer $2 p$.

Call $S_{i}=\partial D_{i}$ and let $\mathscr{S}=\left\{S_{i}\right\}_{i=1}^{r} \cup \mathfrak{A} . \mathscr{S}$ is a tico in $V$. Let $B_{j}^{\prime} \subset B_{j}$ be small closed balls; then $V_{0}=V-\bigcup_{j=1}^{s}$ interior $\left(B_{j}^{\prime}\right)$ is a manifold with spine $\mathscr{S}$, and $\partial V_{0}=\partial V \cup \bigcup_{j=1}^{s} \partial B_{j}^{\prime}, \partial B_{j}^{\prime} \approx S^{m-1}$. See Figure 18. Then we can attach 1-handles to the boundary components of $\partial V_{0}$ and get a manifold $V_{1}=V_{0} \cup$ (1-handles) with $\partial V_{1}=\partial V . V_{1}$ contains the tico $\mathscr{S}$. see Figure 19. 
Let $\left\{C_{j}\right\}_{j=1}^{s}$ be the core circles of the 1-handles; then $|\mathscr{S}| \cup \cup_{j=1}^{s} C_{j}$ is a spine of $V_{1}$. By construction each $C_{j}$ intersects a unique element of $\mathscr{S}$. After an isotopy we can see that $V_{1}$ is obtained by attaching 1-handles to the interior of $V$ (more correctly surgering $S^{0}$ 's in $V$ ). That is, there are closed discs $\left\{B_{l}^{\prime \prime}\right\}_{l=1}^{2 s}$ in $\bigcup_{j=1}^{s} B_{j} \subset V$ such that $V_{1}=\left(V-\operatorname{int} \bigcup_{l=1}^{2 s} B_{l}^{\prime \prime}\right) / \partial B_{2 k-1}^{\prime \prime} \sim \partial B_{2 k}^{\prime \prime}$ for all $k=1,2, \ldots, s$. See Figure 20. Imbed disjoint arcs $\left(I_{k}, \partial I_{k}\right) \hookrightarrow(V \times[1,2), V \times 1)$ and pick tubular neighborhoods $I_{k} \times B^{m}$ of each $I_{k}$ such that $\partial I_{k} \times B^{m}=\left\{B_{2 k-1}^{\prime \prime}, B_{2 k}^{\prime \prime}\right\} \times 1$. Let $Z_{1}=(V \times$ $[0,1]) \cup \bigcup_{k=1}^{s}\left(I_{k} \times B^{m}\right)$ where the union is taken along $\partial I_{k} \times B^{m}$; then $\partial_{+}\left(Z_{1}\right)=V_{1}$. See Figure 21.

Let $N\left(C_{j}\right)$ be a closed tubular neighborhood of the circle $C_{j}$ in $V_{1}$; so there are diffeomorphisms $\theta_{j}: S^{1} \times B^{m-1} \rightarrow N\left(C_{j}\right), j=1, \ldots, s$. Recall $s=2 p$ is an even integer. We can extend these imbeddings to imbeddings

$$
\Theta_{l}:\left(S^{1} \times B^{m-1} \times I, S^{1} \times B^{m-1} \times \partial I\right) \hookrightarrow\left(V \times[0,2)-\operatorname{int}\left(Z_{1}\right), Z_{1}\right)
$$

with $\left.\Theta_{l}\right|_{S^{1} \times B^{m-1} \times \partial I}=\theta_{2 l-1} \amalg \theta_{2 l}, l=1,2, \ldots, p$. In other words we pair up the $N\left(C_{j}\right)$ 's and pairwise identify them by thickened annuli in the complement of $Z_{1}$, i.e. we are attaching round handles to $Z_{1}$ in $V \times[1,2)$. See Figure 22.

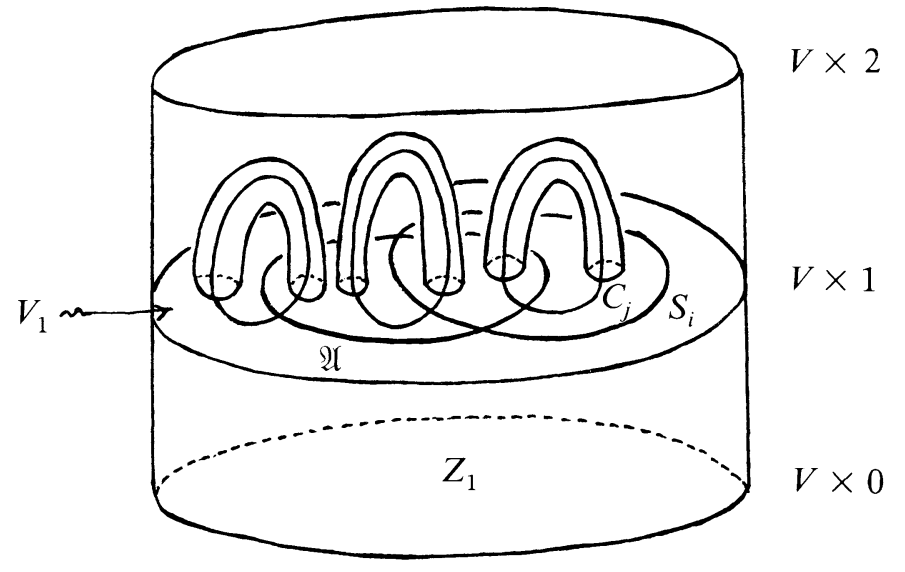

FIGURE 21

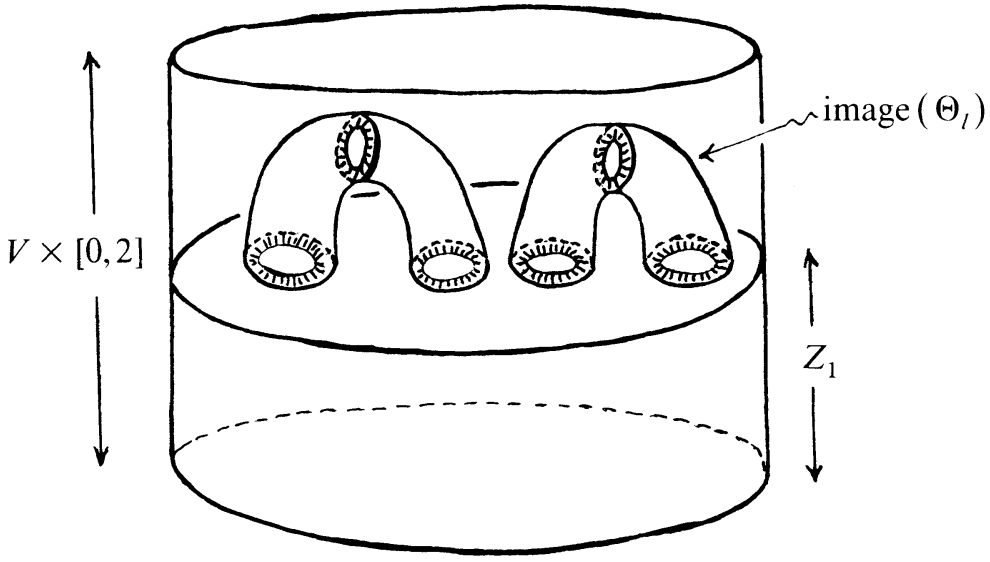

FIGURE 22 


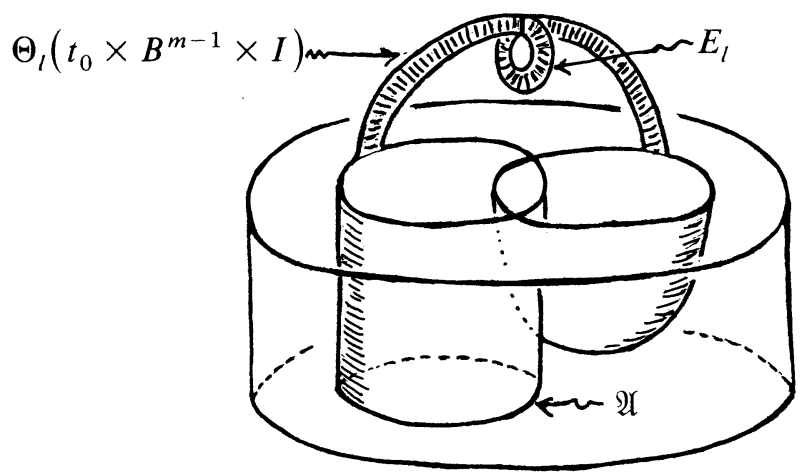

Figure 23

Let $Z_{2}=Z_{1} \cup \cup_{l=1}^{p} \Theta_{l}\left(S^{1} \times B^{m-1} \times I\right)$ where the union identifies $N\left(C_{2 l-1}\right) \cup$ $N\left(C_{2 l}\right)$ in $\partial Z_{1}$ with $\Theta_{l}\left(S^{1} \times B^{m-1} \times \partial I\right)$. Call $\partial_{+}\left(Z_{2}\right)=\bar{V}$. Now define

$$
A=\bigcup_{k=1}^{s}\left(I_{k} \times B^{m}\right) \cup \bigcup_{l=1}^{p} \Theta_{l}\left(S^{1} \times B^{m-1} \times I\right) .
$$

Notice that $\bar{V}=\left(V \times 1-\operatorname{int}\left(\partial_{-} A\right)\right) \cup \partial_{+}(A)$. Next we will define the tico $\mathscr{B}$.

Recall $\mathscr{S}=\left\{S_{i}\right\}_{i=1}^{r} \cup \mathfrak{A}$; write $\mathfrak{U}=\left\{S_{i}\right\}_{i=r+1}^{q}$. Let $\Lambda_{i}=\left\{j \mid C_{j} \cap S_{i} \neq \varnothing\right\}$; then for each $j \in \Lambda_{i}, N\left(C_{j}\right) \cap S_{i}=\theta_{j}\left(t_{0} \times B^{m-1}\right) \approx B^{m-1}$ for some $t_{0} \in S^{1}$.

Define

$$
B_{i}^{\prime}= \begin{cases}\left(D_{i} \times \frac{1}{2}\right) \cup \mathrm{a}\left(S^{i} \times\left[\frac{1}{2}, 1\right]\right) & \text { for } i=1, \ldots, r, \\ S_{i} \times[0,1] & \text { for } i=r+1, \ldots, q .\end{cases}
$$

After smoothing the corners $\left\{B_{i}^{\prime}\right\}$ gives a tico in $V \times[0,1]$. Construct a 1-complex such that vertices are integers $i=1, \ldots, q$ and connect vertices $a$ and $b$ with an edge if $2 l-1 \in \Lambda_{a}$ and $2 l \in \Lambda_{b}$ for some $l=1, \ldots, p$. After reordering we can assume that there are $0=i_{0} \leqslant i_{1} \leqslant \cdots \leqslant i_{\alpha} \leqslant q$ such that the vertices of any connected component of this 1-complex lies in an interval of the form $\alpha_{n}=\left(i_{n}, i_{n+1}\right], n=$ $0,1, \ldots, \alpha$. Let

$$
B_{n}=\bigcup_{i \in \alpha_{n}}\left(B_{i}^{\prime} \cup \bigcup_{2 l \in \Lambda_{i}} \Theta_{l}\left(t_{0} \times B^{m-1} \times I\right)\right), \quad n=0, \ldots, \alpha .
$$

In short, the $B_{n}$ are a union of $B_{i}^{\prime}$ 's which are identified by round handles. Let $E_{l}=\Theta_{l}\left(S^{1} \times B^{m-1} \times \frac{1}{2}\right), l=1, \ldots, p$. Then define $\mathscr{B}=\left\{B_{n}\right\} \cup\left\{E_{l}\right\}$. Then $A$ and $\mathscr{B}$ have the required properties; in particular, $|\mathscr{B}| \cap V \times[0,1]=\bigcup_{i \in \alpha_{n}} B_{i}^{\prime}=$ $|\mathfrak{A}| \times[0,1] \cup\left|\mathscr{B}^{* *}\right|$ where $\mathscr{B}^{* *}$ is a separating tico. See Figure 23.

In particular, we have

$$
\begin{aligned}
& \bar{V}=\left(V_{1}-\bigcup_{l=1}^{p} \operatorname{int}\left(C_{2 l-1}\right) \cup \operatorname{int}\left(C_{2 l}\right)\right) \cup \bigcup_{\partial} \bigcup_{l=1}^{p} \Theta_{l}\left(S^{1} \times S^{m-2} \times I\right), \\
& \mathscr{C}=\left\{\tilde{S}_{n}\right\}_{n=0}^{\alpha} \cup\left\{\Theta_{l}\left(S^{1} \times S^{m-2} \times \frac{1}{2}\right)\right\}_{l=1}^{p},
\end{aligned}
$$


where

$$
\tilde{S}_{n}=\bigcup_{i \in \alpha_{n}}\left[\left(S_{i}-\bigcup_{j \in \Lambda_{i}} N\left(C_{j}\right) \cap S_{i}\right) \cup \bigcup_{2 l \in \Lambda_{i}} \Theta_{l}\left(t_{0} \times S^{m-2} \times I\right)\right] .
$$

COROLLARY 3.2. Let $V^{m}$ be a compact smooth manifold with boundary such that every component of $V$ has nonempty boundary. Let $\mathfrak{A}$ be a closed tico in $V$. Let $\mathfrak{U}^{\prime}$ be a tico in $V^{m}$ such that $\mathfrak{A} \subset \mathfrak{A}^{\prime}$ and $\mathfrak{A}$ is a spine of $\mathfrak{A}^{\prime}$ and if $U$ is any component of $V-\left|\mathfrak{U}^{\prime}\right|$ then $\chi(U) \equiv 0(\bmod 2)$. Then there exists $A$ and $\mathscr{B}$ satisfying the conclusion of Proposition 3.1. Furthermore, they can be chosen so that $A \cap\left|\mathfrak{A}^{\prime}\right| \times[0,2]=\varnothing$, $|\mathscr{E}| \cap\left|\mathfrak{A}^{\prime}-\mathfrak{A}\right| \times[0,2]=\varnothing, \mathscr{C}$ is a spine of $\mathscr{C} \cup\left(\mathfrak{U}^{\prime} \times 1\right)$, and $\mathscr{B}=\mathfrak{A} \times[0,1] \cup \mathscr{B} \mathscr{B}^{\prime}$ where $\mathscr{B}^{\prime} \cap V \times[0,1]=\mathscr{B} * *=\partial_{+} \mathscr{E}$.

Proof. We proceed the same as in the proof of Proposition 3.1 except in the beginning we must choose $\mathscr{D}=\left\{D_{i}\right\}_{i=1}^{r}$ so that it satisfies the additional hypothesis $|\mathscr{D}| \subset V-\left|\mathfrak{A}^{\prime}\right|$. Let $T$ be an open regular neighborhood of $\left|\mathfrak{A}^{\prime}-\mathfrak{U}\right|$ in $V$ and let $T^{\prime}$ be an open collar of $\partial V$. We can cover $V-T \cup T^{\prime}$ by collection of small round closed discs $\mathscr{D}=\left\{D_{i}\right\}_{i=1}^{r}$ whose boundaries are in general position so that $|\mathscr{D}| \cup|\mathfrak{A}|$ is a spine of $V$ and (a) and (b) of properties $\mathscr{D}$ are satisfied. See Figure 24.

The Euler characteristic condition implies, after a similar argument that $U-\cup \partial D_{i}$ is a union of an even number of balls for each $U$, and since $U$ meets the boundary, we can

(i) Choose 1-handles $\left\{I_{k} \times B^{m}\right\}$ connecting components of $U-U \partial D_{i}$, for each $U$.

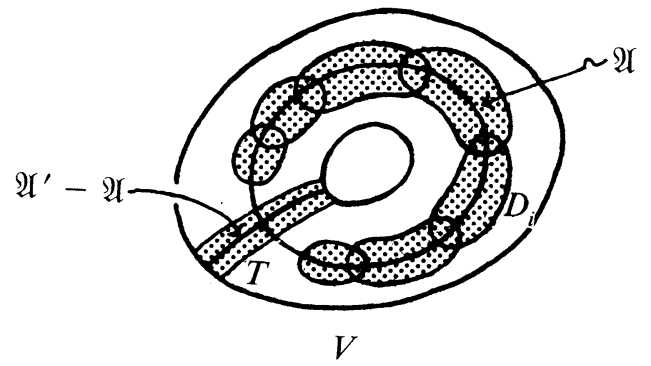

Figure 24

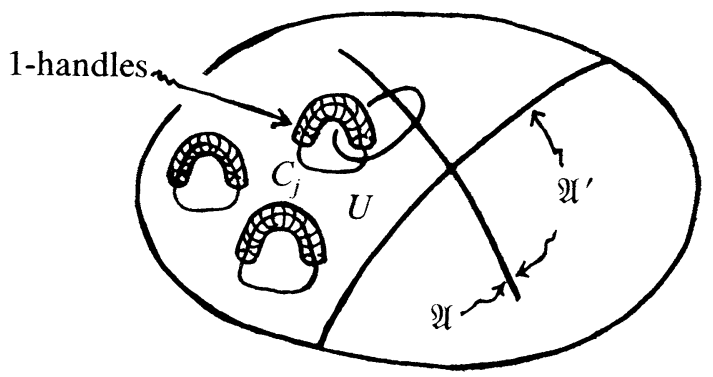

Figure 25 
(ii) Pair up the circles $\left\{C_{j}\right\}$ in each $U \cup$ (1-handles). Then attach the round handles $\left\{\Theta_{l}\right\}$ to these pair of circles, for each $U$.

Hence we can choose (1-handles) $\cup$ (round handles) $=A$ to miss $\mathfrak{A}^{\prime} \times[0,2]$. See Figure 25. By construction $\left|\mathfrak{U}^{\prime} \times 1\right| \cap|\mathscr{C}|=\left|\mathfrak{U}^{\prime} \times 1\right| \cap|\mathfrak{A} \times 1|$, and since $\mathfrak{U}$ is a spine of $\mathfrak{A}^{\prime}$, then $\mathscr{C}$ is a spine of $\mathscr{C} \cup\left(\mathfrak{U}^{\prime} \times 1\right)$.

Proposition 3.3. Let $\mathfrak{F} \in T R$ be seminormal with $V_{0}(\mathfrak{F})=$ a point. Suppose that for some $k \geqslant 1$ and all $0<i<k$ we have diffeomorphisms

$$
h_{i}: \partial V_{i}(\mathfrak{F}) \times(0,1] \rightarrow V_{i}(\mathfrak{F})-V_{0 i}(\mathfrak{F})
$$

so that

(a) $h_{i}\left(\partial V_{j i}(\mathfrak{F}) \times(0,1]\right)=V_{j i}(\mathfrak{F})-V_{0 i}(\mathfrak{F})$ for all $0 \leqslant j<i<k$.

(b) $p_{j i} h_{i}(x, t)=h_{j}\left(p_{j i}(x), t\right)$ for all $(x, t) \in \partial V_{j i}(\mathfrak{F}) \times(0,1], 0<j<i<k$, where $p_{j i}=p_{j i}(\mathfrak{F})$.

Then there is a seminormal $\mathfrak{F}^{\prime} \in T R$ and a diffeomorphism $h_{k}: \partial V_{k}\left(\mathfrak{F}^{\prime}\right) \times(0,1] \rightarrow$ $V_{k}\left(\mathfrak{F}^{\prime}\right)-V_{0 k}\left(\mathfrak{F}^{\prime}\right)$ so that

$\left(\mathrm{a}^{\prime}\right) h_{k}\left(\partial V_{j k}\left(\mathfrak{F}^{\prime}\right) \times(0,1]\right)=V_{j k}\left(\mathfrak{F}^{\prime}\right)-V_{0 k}\left(\mathfrak{F}^{\prime}\right)$ for all $0 \leqslant j<k$,

$\left(\mathrm{b}^{\prime}\right) \quad p_{j k}^{\prime} h_{k}(x, t)=h_{j}\left(p_{j k}^{\prime}(x), t\right)$ for all $(x, t) \in \partial V_{j k}\left(\mathfrak{F}^{\prime}\right) \times[0,1]$ where $p_{j k}^{\prime}=$ $p_{j k}\left(\mathfrak{F}^{\prime}\right)$,

(c') $\mathfrak{F}_{k-1}^{\prime}=\mathfrak{F}_{k-1}$,

$\left(\mathrm{d}^{\prime}\right) \partial \mathfrak{F}^{\prime}=\partial \mathfrak{F}$.

Proof. Let $V_{i}=V_{i}(\mathfrak{F}), \quad V_{j i}=V_{j i}(\mathfrak{F}), \quad \mathfrak{A}_{i}=\mathfrak{A}_{i}(\mathfrak{F}), \quad \mathfrak{A}_{j i}=\mathfrak{A}_{j i}(\mathfrak{F})$, and $p_{j i}=$ $p_{j i}(\mathfrak{F})$. By (a) we know that $V_{i}$ has no closed components for all $0<i<k$. So by Proposition 2.1 we may as well assume that $V_{k}$ has no closed components. If $\operatorname{dim} V_{k}>1$ we may also assume that each connected component $U$ of $V_{k}-\left|\mathfrak{A}_{k}\right|$ has even Euler characteristic. If not, we may use Proposition 2.7 to take the connected sum of $U$ with a torus, thus changing the Euler characteristic of $U$ by one.

Define a map $r:\left|\mathfrak{A}_{k}\right|-V_{0 k} \rightarrow(0,1]$ as follows. Suppose $x \in V_{j k}-V_{0 k}$. Then we let $r(x)=\pi_{j} h_{j}^{-1} p_{j k}(x)$ where $\pi_{j}: V_{j} \times(0,1] \rightarrow(0,1]$ is a projection. By (b) this is well defined since if $x \in V_{j k} \cap V_{i k}-V_{0 k}$ with $0<j<i<k$, then

$$
\pi_{j} h_{j}^{-1} p_{j k}(x)=\pi_{j} h_{j}^{-1} p_{j i} p_{i k}(x)=\pi_{i} h_{i}^{-1} p_{i k}(x) .
$$

Since $\mathfrak{F}$ has type $U$ we know that for each stratum $S$ of $V_{i k}-U_{j<i} V_{j k}, p_{i k} \mid$ : $S \rightarrow V_{i}-\left|\mathfrak{A}_{i}\right|$ is a proper submersion, hence $r \mid: S \rightarrow(0,1]$ is a proper submersion. Hence we may find a compact neighborhood $U$ of $\bigcup_{i=1}^{k-1} V_{i k}$ in $V_{k}$ and a proper submersion $r^{\prime}: U-V_{0 k} \rightarrow(0,1]$ so that $r$ is the restriction of $r^{\prime}$ to $\left|\mathfrak{A}_{k}\right|-V_{0 k}$. What we will do now is to modify $V_{k}$ away from $U$ until $r^{\prime}$ extends to $V_{k}-V_{0 k}$, then use the submersion $r^{\prime}$ to get our promised $h_{k}$.

Notice $r:\left|\mathfrak{A}_{k}\right|-V_{0 k} \rightarrow(0,1]$ is a proper submersion, hence a bundle map, so there is a homeomorphism $g:\left|\partial \mathfrak{A}_{k}\right| \times(0,1] \rightarrow\left|\mathfrak{A}_{k}\right|-V_{0 k}$ so that $\operatorname{rg}(x, t)=t$, so $\mathfrak{A}_{0 k}$ is a spine of $\mathfrak{U}_{k}$.

First let us consider the case where $\operatorname{dim} V_{k}=1$. Then $V_{i k}=\varnothing$ for $0<i<k$ (since $r \mid: V_{I k}-V_{0 k}$ submerses to $(0,1]$ and $\operatorname{dim} V_{i k}=0$, hence $V_{i k}-V_{0 k}=\varnothing$ ). Also by seminormality we know that each component of $V_{k}$ contains at most one point of $V_{0 k}$. If some component of $V_{k}$ contains no points of $V_{0 k}$, we may use 
Proposition 2.7 to connected sum it with a circle with a point tico. Hence we may as well assume that all components of $V_{k}$ contain exactly one point of $V_{0 k}$. It is now easy to define $h_{k}$. Let it be any diffeomorphism $h_{k}: \partial V_{k} \times(0,1] \rightarrow V_{k}-V_{0 k}$; conditions $\left(a^{\prime}\right)$ and $\left(b^{\prime}\right)$ are vacuous.

Now suppose $\operatorname{dim} V_{k}>1$. By Proposition 3.1 and Corollary 3.2 there is a compact codimension zero submanifold $A_{k}$ of $V_{k} \times[1,2)$ and a tico $\mathscr{B}_{k}$ in $V_{k} \times[0,1] \cup A_{k}$ such that if $V_{k}^{\prime}=\left(V_{k} \times 1-\operatorname{int}\left(\partial_{-} A_{k}\right)\right) \cup \partial_{+}\left(A_{k}\right)$ then

(1) $A_{k} \cap\left|\mathfrak{U}_{k}\right| \times[0,2]=\varnothing$,

(2) $\left|\mathscr{B}_{k}\right| \cap V_{k}^{\prime}$ is a spine of $V_{k}^{\prime}$,

(3) $\mathscr{B}_{k}=\mathfrak{U}_{0 k} \times[0,1] \cup \mathscr{B}_{k}^{\prime}$ and $\mathscr{B}_{k}^{\prime} \cap V_{k} \times[0,1]=\partial_{+} \mathscr{D}_{k}$ where $\mathscr{D}_{k}$ is a codimension zero tim.

(4) $\left|\mathscr{D}_{k}\right| \cap\left|\mathfrak{U}_{i k}\right| \times[0,1]=\varnothing$ for all $0<i<k$,

(5) $\left|\mathscr{D}_{k}\right| \cap V_{k} \times 0=\varnothing$ and $\left|\mathscr{D}_{k}\right| \cap \partial V_{k} \times[0,1]=\varnothing$, i.e. $\left|\partial_{-} \mathscr{D}_{k}\right| \subset \operatorname{int}\left(V_{k}\right) \times 1$.

(6) $\mathscr{B} \cap V_{k}^{\prime}$ is a spine of $\mathfrak{A}_{k} \times 1$, in fact $\left|\mathscr{B}_{k} \cap V_{k}^{\prime}\right| \cap\left(\bigcup_{j=1}^{k-1} V_{j k} \times 1\right)=\left(V_{0 k} \cap\right.$ $\left.\bigcup_{j=1}^{k-1} V_{j k}\right) \times 1$.

So by Corollary 2.6 there exists $\mathfrak{F}^{\prime} \in T R$ (which is seminormal by construction) satisfying $\left(\mathrm{c}^{\prime}\right)$ and $\left(\mathrm{d}^{\prime}\right)$. Also $V_{k}\left(\mathfrak{F}^{\prime}\right)=V_{k}^{\prime}, \mathfrak{A}_{j k}\left(\mathfrak{F}^{\prime}\right)=\mathfrak{A}_{j k}(\mathfrak{F}) \times 1$ for $0<j<k$ and $\left|\mathfrak{A}_{0 k}\left(\mathfrak{F}^{\prime}\right)\right|=\left|\mathscr{B}_{k}\right| \cap V_{k}^{\prime}$. Now since $\left|\mathfrak{U}_{0 k}\left(\mathfrak{F}^{\prime}\right)\right|$ is a spine of $V_{k}^{\prime}$ we may extend $r$ to be the proper submersion $r^{\prime \prime}: V_{k}^{\prime}-\left|\mathfrak{A}_{0 k}\left(\mathfrak{F}^{\prime}\right)\right| \rightarrow(0,1]$ so that $r^{\prime} \mid=r$. Let $V_{j k}^{\prime}$ denote $\left|\mathfrak{A}_{j k}\left(\mathfrak{F}^{\prime}\right)\right|$ and let $p_{j k}^{\prime}$ denote $p_{j k}\left(\mathfrak{F}^{\prime}\right)$. Notice $V_{j k}^{\prime}=V_{j k} \times 1$ for $j>0$.

We are now ready to start constructing $h_{k}$. We will construct $h_{k}$ by constructing a vector field $\omega$ on $V_{k}^{\prime}-V_{0 k}^{\prime \prime}$ so that $r_{*}^{\prime \prime}(\omega)=1 ; \omega$ is tangent to the sheets of $\mathfrak{A}_{k}$ and $p_{j k *}^{\prime}(\omega(x))$ is $\partial h_{j}(y, t) / \partial t$ where $y$ is such that $p_{j k}^{\prime}(x)=h_{j}\left(y, t_{0}\right)$ for some $t_{0}$ and $x \in V_{j k}^{\prime}$. We then obtain $h_{k}$ by integrating this vector field. It is easy to construct such a $\omega$ on $\bigcup_{j=1}^{k} V_{j k}^{\prime}-V_{0 k}^{\prime}$, using the fact that $p_{j k}^{\prime}$ submerses all strata to some target stratum (cf. [A $\mathbf{A K}_{4}$, Proposition 2.3]). Just construct $\omega$ stratum by stratum, starting with the lowest dimensional strata. Now extend $\omega$ to all of $V_{k}^{\prime}-V_{0 k}^{\prime}$ and normalize so that $r_{*}^{\prime}(\omega)$ is still 1 .

Repeated applications of Proposition 3.3 give the proof of Theorem 1.2 since the hypotheses (a) and (b) of Proposition 3.3 imply that $\left|\mathfrak{F}_{k-1}\right| \approx$ cone $\left|\partial \mathfrak{F}_{k-1}\right|$ and the conclusions imply $\left|\mathfrak{F}_{k}^{\prime}\right| \approx$ cone $\left|\partial \mathfrak{F}_{k}^{\prime}\right|$.

COROllary 3.4. Theorem 1.2 holds.

4. Obstructions. Let $\mathscr{S}$ denote the set of isomorphism classes of smooth conelike stratified spaces such that each connected stratum has a trivial normal bundle (these are called TCSS spaces in $\left.\left[\mathbf{A K}_{\mathbf{8}}\right]\right)$. Let $\mathscr{S}_{P L}$ denote the set of P.L. homeomorphism classes of elements of $\mathscr{S}$; this means that in $\mathscr{S}_{P L}$ a stratified space is equivalent to a smooth subdivision of itself. Recall that $T R$ is the set (of resolution tower isomorphism classes) of compact resolution towers of type $R S$. Let $T R_{k}$ denote the elements of $T R$ of dimension $\leqslant k$. Define $\eta_{*}^{R}, \eta_{*}^{S}, \eta_{*}^{T}$ to be the cobordism groups of $T R_{*},\left|T R_{*}\right|,\left|T R_{*}\right|_{P L}$ corresponding to the following equivalence relations (1), (2), (3), respectively:

(1) $\mathfrak{\mho}_{1} \sim \mathfrak{F}_{2}$ if there is $\mathfrak{F} \in T R$ with $\partial \mathfrak{\Im}=\mathfrak{\mho}_{1} \amalg \mathfrak{\mho}_{2}$.

(2) $X \sim Y$ if there are $\mathfrak{F}_{1}, \mathfrak{F}_{2}, \mathfrak{F} \in T R$ such that $\partial \mathfrak{F}=\mathfrak{F}_{1} U \mathfrak{F}_{2}$ and $X=\left|\mathfrak{F}_{1}\right|$, $Y=\left|\widetilde{\jmath}_{2}\right|$. 


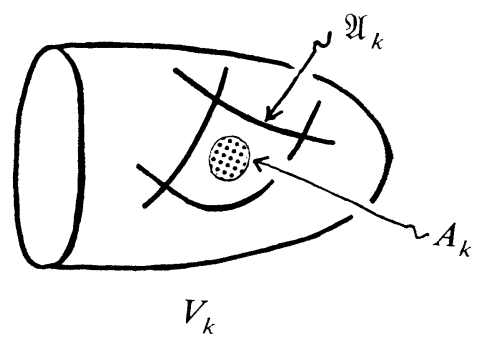

Figure 26

(3) $X \sim Y$ if there are $\mathfrak{F}_{1}, \mathfrak{F}_{2}, \mathfrak{F} \in T R$ such that $\partial \mathfrak{F}=\mathfrak{F}_{1} \amalg \mathfrak{F}_{2}$ and $X^{\prime}=\left|\mathfrak{F}_{1}\right|$, $Y^{\prime}=\left|\mathfrak{F}_{2}\right|$ where $X^{\prime}, Y^{\prime}$ are some smooth subdivisions of $X, Y$.

There are obvious surjections $\eta_{*}^{R} \rightarrow \eta_{*}^{S} \rightarrow \eta_{*}^{T}$. We will denote the cobordism classes by square brackets such as $[\mathfrak{F}],[X]$; it will be clear from the context as to which cobordism group we are referring. It is very likely that if [₹] $=0$ in $\eta_{*}^{R}$ then $\mathfrak{F}=\partial \mathfrak{F}^{\prime}$ for some seminormal $\mathfrak{F}^{\prime} \in T R$, but we are presently unable to prove this. The following proposition gives strong evidence for this.

Proposition 4.1. Let $\mathfrak{F} \in T R_{k}$ with $\mathfrak{F}=\partial \mathfrak{S}$ for some $\mathfrak{G S} \in T R$. Then there exists $\mathfrak{F}^{\prime} \in T R_{k}$ such that $\mathfrak{F}^{\prime}$ and $\mathfrak{F} \amalg \mathfrak{F}^{\prime}$ bound seminormal resolution towers in $T R$.

Proof. Let $\mathbb{S S}=\left\{V_{i}, \mathfrak{A}_{i}, p_{i}\right\}_{i=0}^{n}$. Pick the largest $k$ so that $\mathfrak{G S}_{k-1}$ is seminormal. If $k>n$ we are done. We will prove this by induction on $n-k$. Let $A_{k} \subset \operatorname{int}\left(V_{k}\right)$ $-\left|\mathfrak{A}_{k}\right|$ be a smooth compact codimension zero submanifold so that every component of $V_{k}-\left|\mathfrak{U}_{k}\right|$ meets $\partial V_{k} \cup A_{k}$. See Figure 26.

By Lemma 2.2 there is a compatible family $\left\{A_{i}\right\}_{i=0}^{n}$ in $B S$ with $A_{i}=\varnothing$ for all $i<k$. This gives $\left(S^{\prime \prime}\right.$ and $(S) " \prime \in T R$ where

$$
\begin{aligned}
\mathscr{G S}^{\prime \prime} & =\left\{A_{i}, \mathfrak{A}_{i} \cap A_{i}, p_{i} \mid\right\}_{i=0}^{n} \text { and } \\
\mathfrak{G S S}^{\prime \prime \prime} & =\left\{V_{i}-\operatorname{int}\left(A_{i}\right), \mathfrak{A}_{i} \cap\left(V_{i}-\operatorname{int} A_{i}\right), p_{i} \mid\right\}_{i=0}^{n} .
\end{aligned}
$$

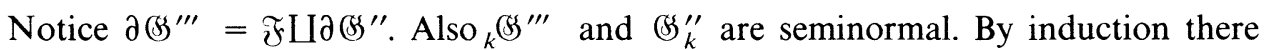
are $\mathfrak{F}^{*}, \mathfrak{F}^{* *} \in T R$ such that $\mathfrak{F}^{*}, \mathfrak{F}^{* *}, \mathfrak{F} \amalg \partial \mathfrak{S}^{\prime \prime} \amalg \mathfrak{F}^{*}, \partial \mathfrak{F}^{\prime \prime} \amalg \mathfrak{F}^{* *}$ all bound seminormal resolution towers. Then $\mathfrak{F}^{\prime}=\partial \mathscr{S}^{\prime \prime} \amalg \mathfrak{F}^{* *} \amalg \mathfrak{F}^{*}$ has the required property.

Hence if we show that bounding seminormal resolution tower is implied by the vanishing of certain additive invariants (characteristic numbers), then Proposition 4.1 would give that if $\mathfrak{F}$ bounds, then it bounds a seminormal resolution tower. This holds for resolution towers of dimension $\leqslant 2\left[\mathbf{A K}_{\mathbf{5}}\right]$, so we conjecture

CONJECTURE (*). If $\mathfrak{F} \in T R$ bounds, then it bounds a seminormal resolution tower.

The positive answer to this conjecture would give a nicer obstruction theory than what we will describe in this paper. To give the reader some idea here we give the values of some of these cobordism groups ( 
TABLE 1

\begin{tabular}{c|c|c|c|} 
& $i=0$ & $i=1$ & $i=2$ \\
\hline$\eta_{i}^{T}$ & $\mathbb{Z} / 2 \mathbb{Z}$ & $\mathbb{Z} / 2 \mathbb{Z}$ & $(\mathbb{Z} / 2 \mathbb{Z})^{5}$ \\
\hline$\eta_{i}^{S}$ & $\mathbb{Z} / 2 \mathbb{Z}$ & $(\mathbb{Z} / 2 \mathbb{Z})^{2}$ & $(\mathbb{Z} / 2 \mathbb{Z})^{15}$ \\
\hline$\eta_{i}^{R}$ & $\mathbb{Z} / 2 \mathbb{Z}$ & $(\mathbb{Z} / 2 \mathbb{Z})^{2}$ & $(\mathbb{Z} / 2 \mathbb{Z})^{65}$ \\
\hline
\end{tabular}

Let $\mathscr{S}_{n}$ denote the elements of $\mathscr{S}$ of dimension $\leqslant n$. Let $Y \in \mathscr{S}_{n}$ and let $Z$ be an $s$-dimensional substratified set in $Y$. A $T R$-structure on $Y-Z$ is an $\mathfrak{F} \in T R$ such that $|\mathfrak{F}|=Y-Z$; we further demand that $|\mathfrak{F}|$ restricts to a product structure on a tubular neighborhood of each connected stratum of $Y-Z$ and on the boundary of the tubular neighborhood of each $s$-dimensional connected stratum of $Z$. Now fix a $T R$-structure on $Y-Z$. Let $\sigma$ be an open connected $s$-dimensional stratum of $Z$ and let $Z^{\prime}=Z-\sigma$. Then the boundary of the tubular neighborhood of $\sigma$ is in the form $|\mathfrak{F}| \times \sigma$ for some $\mathfrak{F} \in T R_{n-s-1}$. Then we have

THEOREM 4.2. The TR-structure of $Y-Z$ extends to $Y-Z^{\prime}$ if and only if there is a seminormal $\mathbb{B S} \in T R$ such that $\mathfrak{F}=\partial$ (S).

ProOF. If the structure extends across we would have a $T R$-structure on the tubular neighborhood cone $|\mathfrak{F}| \times \sigma$ of $\sigma$ restricting to the old structure $|\mathfrak{F}| \times \sigma$ on the boundary. Hence there exists $\mathscr{S}$ with $\partial \mathscr{G}=\mathfrak{F}$ and $|\mathfrak{G}|=\operatorname{cone}|\mathfrak{F}|$ so that $\mathscr{G} \times \sigma$ is the extension. In particular, $\mathscr{G}$ is seminormal. Conversely if $\mathfrak{F}=\partial \mathfrak{S}$ for some seminormal $\mathscr{S}$, by Theorem 1.2 there is $\mathscr{S S}^{\prime}$ with $\partial \mathscr{S S}^{\prime}=\partial \mathscr{S S}^{\prime}$ and $\left|\mathscr{S S}^{\prime}\right|=\operatorname{cone}|\partial \mathscr{S}|=$ cone $|\mathfrak{F}|$. Hence $\mathscr{B S}^{\prime} \times \sigma$ gives the required extension.

Now triangulate $Y$, let $X$ and $\Sigma$ be the triangulated copies of $Y$ and $Z$, respectively. $\operatorname{Recall} \operatorname{dim}(\Sigma)=s$. In particular, the boundary of the tubular neighborhood of each open $s$-simplex of $\Sigma$ is in the form $|\mathfrak{F}| \times \mathbf{R}^{s}$ for some $\mathfrak{F} \in T R_{n-s-1}$. That is, the link of each $s$-simplex has a $T R$-structure $|\mathfrak{F}|$. Let $\xi(\Sigma)$ be the formal sum of all $s$-simplexes of $\Sigma$ with these coefficients in $T R_{n-s-1}$. In other words, if $\left\{\sigma_{i}\right\}_{i=0}^{k}$ is the set of all s-simplexes, $\xi(\Sigma)=\sum_{i=0}^{k} \sigma_{i} \mathfrak{F}_{i}$ where $\left|\mathfrak{F}_{i}\right|$ is the link of $\sigma_{i}$. Let $\Sigma^{\prime}=\Sigma-\bigcup_{i=0}^{k} \operatorname{int}\left(\sigma_{i}\right)$; we call $\xi(\Sigma)$ the total obstruction to extending the structure to $X-\Sigma^{\prime}$. Theorem 4.2 say that the $T R$-structure on $X-\Sigma$ extends to $X-\Sigma^{\prime}$ if and only if each $\mathfrak{F}_{i}$ bounds a seminormal resolution tower. If we allow changing the $T R$-structure on $X-\Sigma$ before extending, we get weaker obstructions to extension as follows. First we need to define the connected sum operation. If $\mathfrak{F}, \mathfrak{F}^{\prime} \in T R$ with $\operatorname{dim}(\mathfrak{F})=\operatorname{dim}\left(\mathfrak{F}^{\prime}\right)=m$, we can form a new resolution tower $\mathfrak{F} \# \mathfrak{F}^{\prime}$ by

$$
V_{i}\left(\mathfrak{F} \# \mathfrak{F}^{\prime}\right)= \begin{cases}V_{i}(\mathfrak{F}) \amalg V_{i}\left(\mathfrak{F}^{\prime}\right) & \text { for } i<k, \\ V_{m}(\mathfrak{F}) \# V_{m}\left(\mathfrak{F}^{\prime}\right) & \text { for } i=m,\end{cases}
$$


where the connected sum is taken along discs lying in the interiors of $V_{m}(\mathfrak{F})$ $\left|\mathfrak{U}_{m}(\mathfrak{F})\right|$ and $V_{m}\left(\mathfrak{F}^{\prime}\right)-\left|\mathfrak{A}_{m}\left(\mathfrak{F}^{\prime}\right)\right|$, and $p_{i}\left(\mathfrak{F} \# \mathfrak{F}^{\prime}\right)$ defined by restriction. Now let $(X, \Sigma)$ be as above and let $\Delta$ be any $(s+1)$-simplex of $X$ with $\partial \Delta \subset \Sigma$. Let $\partial \Delta=\sum_{i=0}^{s+1} \sigma_{i}$, and let the dimension of the link of $\Delta$ be $r-1$ (i.e. the local dimension of $X$ near $\Delta$ is $s+1+r$ ). Then the following proposition says that we have some freedom of changing the total obstruction near $\partial \Delta$.

Proposition 4.3. Given a TR-structure on $X-\Sigma$ with the obstruction $\xi(\Sigma)=$ $\sum_{i=0}^{k} \sigma_{i} \mathfrak{F}_{i}$ and $a \mathbb{S} \in T R_{r}$ with $|\mathfrak{S}| \underset{P L}{\approx} S^{r}$, then after subdividing $X-\Sigma$ we can change the TR-structure near $\Delta$ such that the new TR-structure on $X-\Sigma$ has the obstruction $\xi^{\prime}(\Sigma)=\sum_{i=0}^{s+1} \sigma_{i}\left(\mathfrak{\mho}_{i} \#(\mathfrak{S})+\sum_{i>s+1}^{k} \sigma_{i} \widetilde{\mho}_{i}\right.$.

Proof. Let $|\mathfrak{\mho}|$ be the link of $\Delta$. We have $\operatorname{dim}|\mathfrak{F}|=r-1$. Recall $\left|\mathfrak{F}_{i}\right|$ are the links of $\sigma_{i}, i=0, \ldots, k$. Let $U$ denote the tubular neighborhood of $\operatorname{int}(\Delta)$. $U$ has a $T R$-structure by assumption so there is $\mathfrak{F}^{\prime} \in T R_{r}$ with $\partial \mathfrak{F}^{\prime}=\mathfrak{F}$ and $U=$ $\left|\mathfrak{F}^{\prime}\right| \times \operatorname{int}(\Delta)$. Let $\mathfrak{F}^{\prime \prime}=\mathfrak{F}^{\prime} \#(\mathfrak{G}$, i.e.

$$
V_{i}\left(\mathfrak{F}^{\prime \prime}\right)= \begin{cases}V_{i}\left(\mathfrak{F}^{\prime}\right) \amalg V_{i}(\mathfrak{G}) & \text { for } i<r, \\ V_{r}\left(\mathfrak{F}^{\prime}\right) \# V_{r}(\mathfrak{G S}) & \text { for } i=r .\end{cases}
$$

Notice $\mathfrak{F}^{\prime}$ is a codimension zero subtower of each $\mathfrak{F}_{i}$ for $i=0, \ldots, s+1$. So changing the structure on $U$ changes the links of each $\sigma_{i}$ to $\left|\mathfrak{F}^{k} \# \mathbb{G}\right|$ for $i=0, \ldots, s$ +1 leaves the links of $\sigma_{i}$ for $s+1<i \leqslant k$ unchanged. See Figure 27.

Now assume that $X$ has a constant local dimension. This means that the dimension of the link of each $i$-simplex is $n-i-1$. Also assume that $\Sigma$ is the $s$-skeleton of $X$. Let $\tilde{\xi}(\Sigma)$ be the chain $\sum_{i=0}^{k} \sigma_{i}\left[\mathfrak{\mho}_{i}\right]$ in $C_{s}\left(X ; \eta_{n-s-1}^{R}\right)$. If Conjecture (*) holds then vanishing of $\tilde{\xi}(\Sigma)=0$ would imply that the $T R$-structure on $X-\Sigma$ extends to $X-\Sigma^{\prime}$. We claim that $\tilde{\xi}(\Sigma)$ is a cycle, i.e. $\partial_{s} \tilde{\xi}(\Sigma)=0$ where $\partial_{s}: C_{s}\left(X ; \eta_{n-s-1}^{R}\right) \rightarrow C_{s-1}\left(X ; \eta_{n-s-1}^{R}\right)$ is the boundary map. This follows by looking at the link of each $(s-1)$-dimensional simplex of $\Sigma$; then the part of the link which lies in the complement of $\Sigma$ gives the cobordism needed to have $\partial_{s} \tilde{\xi}(\Sigma)=0$. See Figure 28.

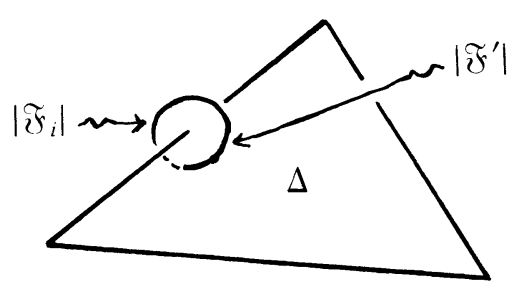

FIGURE 27 


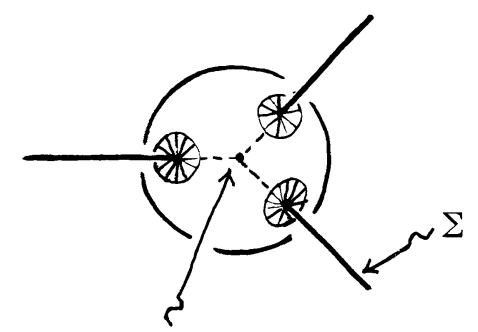

an $(s-1)$-simplex of $\Sigma$

\section{FigURE 28}

So $\tilde{\xi}(\Sigma) \in \operatorname{ker} \partial_{s}$. Let $\theta_{k}$ be the subgroup of $\eta_{k}^{R}$ generated by resolution towers (s) with $\mid$ SS $\mid$ P.L. homeomorphic to $S^{k}$. There is a natural map $\rho_{s+1}: C_{s+1}\left(X ; \theta_{n-s-1}^{R}\right) \rightarrow$ $C_{s+1}\left(X ; \eta_{n-s-1}^{R}\right)$ induced by inclusion, let $\bar{\partial}_{s+1}=\partial_{s+1} \circ \rho_{s+1}$. Proposition 4.3 says that by adjusting the $T R$-structure on $X-\Sigma$ we can change $\tilde{\xi}(\Sigma)$ by the image of $\bar{\partial}_{s+1}$. Define $\theta_{s}(X)=\operatorname{kernel}\left(\partial_{s}\right) /$ image $\left(\bar{\partial}_{s+1}\right)$. Let $\xi^{*}(\Sigma) \in \theta_{s}(X)$ be the image of $\tilde{\xi}(\Sigma)$; then we can summarize the preceding discussion as follows.

COROLlary 4.4. If Conjecture (*) holds, then (a) $\tilde{\xi}(\Sigma)=0$ if and only if the $T R$-structure on $X-\Sigma$ extends to $X-\Sigma^{\prime}$.

(b) If $\xi^{*}(\Sigma)=0$, then after possibly changing the structure near the $(s+1)$-skeleton and subdividing $X-\Sigma$ the structure on $X-\Sigma$ extends to $X-\Sigma^{\prime}$.

\section{REFERENCES}

$\left[\mathbf{A K}_{1}\right]$ S. Akbulut and H. King, The topology of real algebraic sets, L'Enseignement Math. 29 (1983), 221-261.

$\left[\mathbf{A K}_{2}\right] \ldots$, Resolution tower.

$\left[\mathbf{A K}_{3}\right]_{\ldots} \ldots$, Resolution towers on real algebraic sets.

$\left[\mathbf{A K}_{\mathbf{4}}\right] \ldots$, Algebraic structures on resolution towers.

$\left[\mathbf{A K}_{5}\right] \ldots$, The topological classification of 3-dimensional real algebraic sets.

$\left[\mathbf{A K}_{\mathbf{6}}\right]$ _, The topologv of real algebraic sets with isolated singularities, Ann. of Math. 113 (1981), $425-446$.

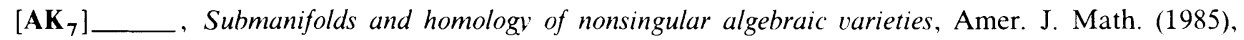
45-83.

[A $\left.\mathbf{A K}_{\mathbf{8}}\right]_{\ldots}$, Real algebraic structures on topological spaces, Publ. Math. Inst. Hautes Etudes Sci. 53 (1981), 79-162.

[AT] S. Akbulut and L. Taylor, A topological resolution theorem, Publ. Math. Inst. Hautes Etudes Sci. 53 (1981), 163-195.

[D] D. Sullivan, Singularities in spaces, Proc. Liverpool Singularities II, Lectures Notes in Math., vol. 209, Springer-Verlag, Berlin and New York, 1971, pp. 196-206.

Department of Mathematics, Michigan State University, East Lansing, Michigan 48824

Department of Mathematics, University of Maryland, College Park, Maryland 20742 